\title{
Pratiques
}

Linguistique, littérature, didactique

\section{Écrire à partir d'une image et fabriquer un objet à partir d'un texte : historique et caractéristiques actuelles des pratiques scolaires interartiales}

Writing from an image and fabricating an object from a text: history and current characteristics of interartial school practices

\section{Nicole Biagioli}

\section{CpenEdition}

Journals

Édition électronique

URL : http://journals.openedition.org/pratiques/3587

DOI : $10.4000 /$ pratiques.3587

ISSN : 2425-2042

Éditeur

Centre de recherche sur les médiations (CREM)

\section{Référence électronique}

Nicole Biagioli, «Écrire à partir d'une image et fabriquer un objet à partir d'un texte : historique et caractéristiques actuelles des pratiques scolaires interartiales », Pratiques [En ligne], 175-176 | 2017, mis en ligne le 22 décembre 2017, consulté le 14 novembre 2019. URL : http://

journals.openedition.org/pratiques/3587; DOI : 10.4000/pratiques.3587

Ce document a été généré automatiquement le 14 novembre 2019.

(C) Tous droits réservés 


\title{
Écrire à partir d'une image et fabriquer un objet à partir d'un texte : historique et caractéristiques actuelles des pratiques scolaires interartiales
}

\author{
Writing from an image and fabricating an object from a text: history and \\ current characteristics of interartial school practices
}

Nicole Biagioli

\section{Introduction}

Suite aux réformes scolaires qui de 2008 à 2015 ont mis l'accent en France sur l'enseignement-apprentissage des rapports entre la littérature et les arts, des études récentes se sont penchées sur la compétence de compréhension des œuvres littéraires et artistiques développée par les élèves, en comparant les deux approches, littéraire et artistique (Claude, 2016), ou en montrant en quoi l'une - le commentaire de l'art - peut servir l'autre - l'enseignement de la langue et de la littérature (Chabanne, 2016). Nous avons choisi d'opérer un déplacement de la focale sur la compétence de production, et sur l'entrecroisement des pratiques artistiques qui constitue le champ de l'interartialité ${ }^{1}$. Nous y avons été incitée par l'importance prise par l'interartialité dans les arts contemporains, notamment la littérature. La littérature contemporaine a modifié simultanément la conception des disciplines artistiques et la place du sujet qu'elle met «dans une posture critique au cœur de l'interaction entre diverses modalités sémiotiques" (Bricco \& Murzilli, 2012, p. 2). Et l'ouverture à l'interartialité est «le propre d'une littérature inquiète, qui interroge l'existence et le monde avec la conscience critique de l'incontournabilité d'une médiation, dont elle fait elle-même le choix, pour accéder à la compréhension des êtres et des choses" (ibid.). Si l'on veut 
familiariser la communauté scolaire avec ce changement culturel, il faut d'abord en mesurer le cout en termes de modification des représentations, des habitudes et des pratiques. En tant qu'organe de transmission, l'école est en décalage avec les savoirs et pratiques en émergence. C'est aussi pour cela qu'au fur et à mesure que les apprentissages se complexifient, les disciplines se cloisonnent. L'aggiornamento s'annonce donc difficile.

2 Nous avons fait l'hypothèse que l'enseignement-apprentissage de la production artistique interartiale était une voie d'initiation à l'interartialité plus directe que la décontextualisation-recontextualisation de connaissances culturelles hétérogènes ou aléatoires. Deux faits nous y ont encouragée. D'une part, cet enseignementapprentissage existe déjà, aux États-Unis, depuis que les formations de creative writing ont migré des départements de littérature des universités vers les écoles d'art et que, d'après le témoignage d'un enseignant d'art, les étudiants futurs écrivains « réalisent que les questions qui sont à la base du creative writing sont les mêmes que celles qui sont à la base de la peinture ou des autres arts : questions d'équilibre, de dynamisme, etc. qui nous sont familières pour les arts plastiques " (Swensen, cité par Oriol-Boyer \& Duminy-Sauzeau, 2016, p. 593).

D'autre part, il existe dans la culture scolaire, et notamment dans l'histoire de la discipline français, une longue pratique de la mise en synergie de l'image et du texte. M. Butor (1969), découvrant l'omniprésence des mots dans la peinture occidentale, a pu rendre responsable de son aveuglement «le mur fondamental édifié par notre enseignement entre les lettres et les arts » (quatrième de couverture des Mots dans la peinture). Mais une enquête un peu approfondie (Biagioli, 2013) sur les circonstances de cette édification fait apparaitre à la fois la nécessité de la séparation de l'écrit et de l'image, pour des raisons d'apprentissage, et, pour les mêmes raisons, celle de leur collaboration dans l'étayage de la lecture, depuis l'abécédaire jusqu'à l'explication de texte.

4 Ceci pointe une difficulté tout autant technique que théorique : la distinction entre intermédialité et interartialité, la seconde étant une spécification de la première (Moser, 2007). Celle-ci n'est pas toujours aisée à faire, en particulier dans le contexte scolaire. En effet, à l'école, littérature et art n'apparaissent pas immédiatement comme tels, mais d'abord à travers la matière qui les constitue : leur média, et l'apprentissage que celui-ci requiert. Cet apprentissage suppose à la fois une spécialisation - on apprend à lire d'un côté, à dessiner ou à chanter de l'autre -, et une familiarisation avec la combinaison des médias dans les messages intermédiaux, qui ne sont pas tous artistiques mais peuvent le devenir (ex: les cartes de géographie insérées dans un roman). Autre filtre scolaire: la désartialisation de l'art. Enseignée, l'œuvre devient outil de sa propre connaissance. On ne la lit pas, on la « décortique ».

5 Il y a donc au moins deux raisons pour qu'une œuvre interartiale, par exemple un opéra, soit désartialisée. Elle n'est là que pour l'apprentissage de sa catégorie générique. Et il faut que par-delà le but, on redécouvre les moyens de sa production : le tissage intermédial de plusieurs sémiotiques (pour l'opéra, le théâtre, déjà mixte de texte et de jeu, et la musique). Nous conserverons toutefois le terme "interartialité " pour désigner l'objectif didactique, même si l'apprentissage traite parfois l'intermédialité sans l'actualiser dans une situation explicitement artistique.

6 Nous avons organisé notre étude en deux parties. Dans la première, nous retraçons brièvement les étapes-clefs de la didactisation de l'interartialité, depuis 1975 jusqu'aux 
réformes récentes, en nous aidant des manuels que nous considérons comme des témoignages fiables non des pratiques enseignantes mais des problématiques d'adaptation des objectifs des programmes aux réalités culturelles de l'école et aux réalités économiques du marché de l'édition scolaire. La seconde partie sera consacrée à l'analyse des résultats de deux enquêtes réalisées, l'une sur la production d'un écrit littéraire à partir d'une première de couverture de roman, l'autre sur la réalisation d'un objet pour rendre compte d'une lecture cursive faite à la maison. Nous avons centré notre étude sur le collège, parce que le seuil primaire-secondaire marque, du moins en France, le passage à des enseignements disciplinaires explicitement cloisonnés et pose le problème de leur mise en cohérence. Enfin, il concerne une tranche d'âge dans sa totalité et reste le lieu « unique », et donc la pierre de touche, de la démocratisation de l'enseignement.

\section{L'interartialité dans les manuels de collège}

7 Deux remarques pour introduire cette partie. D'abord sur l'enjeu de la création du collège unique qui continue d'être un objet de débat national en France. En mettant en continuité deux types d'enseignement auparavant parallèles, il a fait se rencontrer des univers sociaux que seules les guerres avaient auparavant réunis. Ces univers relevaient de cultures très différentes, et, si les possesseurs de la culture légitime aimaient s'encanailler au café concert ou dans les fêtes paysannes, les vrais lieux de rencontre culturelle étaient rares. De nos jours, ce clivage s'est complexifié et déplacé (par exemple, l'opéra parait plutôt rattaché à la culture légitime qu'à la culture populaire), mais le principe demeure: les privilégiés, dans le public comme chez les artistes, sont ceux qui peuvent passer d'un registre culturel à un autre à leur gré. Il y a donc pour les manuels scolaires une sorte de clause d'inclusion culturelle. Ils doivent rappeler l'ensemble des codes artistiques et des conduites esthétiques présents dans la société, et les mettre en perspective réciproque et collective.

8 L'autre remarque concerne l'évolution des médias et son incidence sur le contexte des apprentissages. Les progrès des techniques de reproduction couleur, l'image numérique et enfin le passage au manuel électronique ont totalement modifié la physionomie des manuels, en accroissant l'effet de sidération pan-iconique, mais aussi en articulant de façon beaucoup plus efficace que les injonctions des chercheurs en éducation, l'univers professionnel et l'univers personnel des acteurs scolaires.

\section{L'introduction de l'image dans les programmes de français du collège unique (1977-1995)}

Le traitement de l'image et des arts dans les manuels durant les premières années de l'existence du collège unique manifeste ce qui va devenir une constante : tous les arts passent par la reproduction iconique d'où l'ambiguïté sémiotique de la désignation qui fait comme si c'était l'œuvre que l'on voyait. S'y mêlent également les illustrations propres au manuel. Outre la diversité des statuts, celle des fonctions contribue à brouiller la nature de l'image. À cette époque, elle n'est pas étudiée pour elle-même, mais toujours en rapport avec un texte, même si l'on fournit les éléments d'information qui permettent de l'identifier.

10 Les instructions de 1977 ont une triple orientation : 
on reprend à la fois la tradition du primaire, centrée sur la culture pratique, en développant les qualités d'observation et de description du réel, et celle du secondaire, invitant à écrire à partir de l'étude des textes littéraires ; enfin émerge le souci de développer l'imagination, en relation avec une nouvelle conception de la littérature, plus sensible qu'auparavant à la fiction. (Boissinot \& Houdart-Merot, 2001, p. 37)

11 L'image est bi-culturelle mais elle est aussi bi-fonctionnelle: elle étaye la compréhension et la production écrites et orales. Cette double mission fait d'elle le pivot de «la trilogie fondamentale : écrit, oral, image » (ibid.), orientant son emploi vers l'intermédialité plus que l'interartialité, et restreignant celle-ci au rapport littérature-peinture héritée de l'iconographie des manuels de lycée. Exemple canonique de cette interartialité restreinte : l'exploitation dans le XVII ${ }^{e}$ siècle (Lagarde \& Michard, 1967) des Bergers d'Arcadie de Poussin en couverture puis en double page pour introduire le théâtre de Corneille ${ }^{2}$. Enfin, corrélé à "imagination », le mot "image » renvoie indifféremment à l'image mentale et à l'image réelle, une ambiguïté entretenue à dessein qui fait de l'image sous ses deux espèces la clef unique de la compréhension et de la création.

Pour illustrer cette période, nous avons choisi le Mots et Merveilles $5^{e}$ (Arnaud \& Garrigue, 1982, p.2) dont les auteurs précisent: «Les illustrations ont été choisies comme échos, ouvertures ou contrepoints aux textes et peuvent être sources de multiples travaux oraux et/ou écrits ". On retrouve donc bien l'axe intermédial dans la visée illustrative et la relation à l'oral et à l'écrit, et les potentialités interartiales dans les rapports aux textes proposés. Deux éléments cependant restent à préciser: l'artialité de l'image et du texte est-elle explicite ? Et s'il ne s'agit pas de textes ou d'image artistiques, l'activité de compréhension ou de production qui est menée estelle de nature esthétique?

Rappelons en effet que ce n'est pas le message qui fonde l'expérience esthétique, mais le type d'attention qu'on lui accorde. Nous reprenons donc à notre compte l'argumentation de J.-M. Schaeffer (2015, p. 52) :

Les opposants de la notion d'«attitude esthétique» ont donc raison quand ils soutiennent que l'attention esthétique utilise les ressources génériques de l'attention, mais ils ont tort lorsqu'ils en concluent qu'elle n'existe pas en tant que mode spécifique, parce qu'ils omettent d'envisager la possibilité que les mêmes ressources peuvent être engagées dans des stratégies attentionnelles différentes.

14 Elle est particulièrement adaptée au traitement de deux problématiques qui sont au cœur de l'enseignement-apprentissage de la posture esthétique : celle de la relation entre les codes sociaux répercutés par l'école et le gout et le capital culturel des individus, et celle du rapport entre la compréhension et la production dans l'expérience esthétique.

Précisons que l'interartialité comporte plusieurs régimes, condition qui va également peser sur sa didactisation. Elle peut être pratiquée par le même individu qui s'exprime en passant d'un art à l'autre ou en les mélangeant (cas de V. Hugo, J. Cocteau, R. Desnos, H. Michaux, tous auteurs que nous allons retrouver dans les manuels, sans que l'on signale forcément cette particularité, ou si on la signale, sans qu'on l'exploite). Elle peut être réalisée par la collaboration entre artistes de disciplines différentes, et là aussi, de deux façons, soit en décalé, soit en synergie, ce qui par exemple donnera dans le premier cas le livre illustré (l'illustrateur intervenant indépendamment de l'auteur et souvent de façon posthume) et dans le second, le livre d'artistes (Giraudo, 2006, p. 71). 
La collaboration peut également être suscitée par un projet qui n'émane pas directement des artistes mais d'un commanditaire, souverain ou mécène, c'est alors lui qui réunit les artistes et favorise les échanges.

Sans surprise, la littérature et la peinture sont les arts les mieux identifiés. Le choix iconographique tient compte de l'actualité picturale: street art à Paris (Arnaud \& Garrigue, 1982, p. 167), à Rome (ibid., p. 147), confronté avec une fresque d'Ernest Pignon-Ernest à Paris (ibid., p. 146); de la précision historique. La représentation des genres picturaux (scène d'intérieur de Vermeer, paysage de Corot), et des genres littéraires (poésie, théâtre, roman) est équilibrée sans toutefois donner lieu à une mise en parallèle. Les deux présentations explicites de tableaux (avec questionnaire image) concernent l'une, un impressionniste (Van Gogh), l'autre un symboliste (Redon). Pour la peinture, le recours à l'imprégnation reste dominant, ce qui n'est pas le cas pour la littérature. Les deux arts sont bien identifiés, prérequis de l'approche interartiale, mais celle-ci n'est pas explicitée lorsqu'elle se rencontre sous sa forme auctoriale. La reproduction d'une signature de $\mathrm{V}$. Hugo en lettres décoratives entrelaçant son prénom avec «Jersey » et son nom avec une chaine de bagnard, sous un extrait des Châtiments, ne donne lieu à aucun commentaire.

La forme interauctoriale, collaboration entre artistes de disciplines différentes, est, elle, très présente. Héritage de l'enseignement primaire, elle passe essentiellement par la pratique. On demande d'illustrer Le dormeur du val d'A. Rimbaud, en "utilisant seulement des taches de couleur en harmonie avec les sentiments qu'éveillent en vous ces vers" (ibid., p. 20), ce qui montre un souci à la fois de familiariser avec un trait stylistique et de s'adapter aux possibilités de l'élève. On propose, suite à la « lecture d'un vitrail» de Chartres qui retrace des épisodes de la chanson de Roland, de "transposer, selon la technique du vitrail un autre épisode de la Chanson de Roland " (ibid., p. 57), en fournissant le schéma général de la scène centrale entourée de scènes secondaires. Bien sûr, l'élève n'accède qu'à un stade préparatoire de l'exécution, mais on voit bien ce que cela permet: une relecture de l'épopée, débouchant sur une hiérarchisation spatiale, différente de celle de la séquence narrative, et un apprentissage complet, sinon exhaustif, de la communication interartiale.

Pourquoi alors n'avoir pas proposé aux élèves d'écrire leur nom en lettres décoratives, à l'instar de V. Hugo ? C'est là un autre intérêt de la stratégie didactique de ce manuel. Il s'efforce de ne pas limiter l'interartialité au compagnonnage de la littérature et des arts visuels. D'une part, il équilibre le partage intermédial du trait entre le texte et l'image par celui du son entre le texte et la musique, en s'appuyant par exemple sur les $1^{\mathrm{er}}$ et $4^{\mathrm{e}}$ mouvements de la Symphonie Pastorale de Beethoven, que l'élève doit intituler, réécouter, avant de produire un texte libre, puis de dessiner ou peindre son ressenti (ibid., p. 197). Mais il part aussi des productions d'élèves. Une lecture chorale (ibid., p. 306) commence par une improvisation vocale enregistrée, qui suggère des personnages, actualisés par le mime ou la fabrication de marionnettes, lesquels vont donner lieu à l'invention d'un scénario, sur lequel on improvise un dialogue chanté (une sorte de comédie musicale), avec accompagnement également improvisé de percussions ou d'autres instruments.

19 Il convoque également d'autres arts que le dessin et la musique, et donc d'autres disciplines que le français, la musique et les arts plastiques. La construction des marionnettes implique la technologie, le mime pour comprendre un texte qui décrit le travail à la chaine, l'EPS. Enfin, d'autres genres, qui ne sont pas artistiques, apportent 
un contrepoint critique à l'étude de la fiction. À l'issue d'un dossier qui confronte les stéréotypes de l'Indien dans le western et la BD avec une étude ethnologique sur les danses des Indiens d'Amérique, on demande aux élèves: "Avec le professeur d'EPS, mimez la chasse. Ensuite rédigez un texte libre que vous illustrerez à votre gré, pour leur proposer ensuite comme « jeu dramatique une parodie de western » (ibid., p. 115).

Cette frénésie interartiale s'explique par la volonté de faire découvrir les invariants structurels des langages artistiques. Elle repose aussi sur la conviction que l'élève doit être impliqué pour comprendre, et que la mise à distance du ressenti passe par la pratique, puisque celle-ci implique un retour sur le ressenti, ne serait-ce que pour le faire partager. Enfin, elle garde le souvenir des apprentissages premiers qui s'appuient sur le geste et la manipulation pour faciliter la représentation et la compréhension.

Rappelons que nombre des premiers enseignants de collège étaient d'anciens enseignants du primaire, formés à la polyvalence, la pédagogie de projet et les travaux de groupe, adeptes aussi parfois de la pédagogie Freinet du « texte libre ».

Si, dans ce manuel, l'interartialité prévaut sur l'intermédialité - on est très loin de la simple trilogie écrit, oral, image - c'est à la faveur d'un engagement éthique autant qu'artistique qui rejoint le souhait de M. Butor «d'une nouvelle distribution des activités humaines, d'un art qui soit science en même temps, d'une littérature qui soit en même temps quelque peu musique et peinture " (cité par Giraudo, 2006, p. 22). À preuve la conclusion de la présentation: "Médiateur, le professeur de français doit rester celui qui accueille, comprend, éveille, guide pour que les mots conduisent les enfants au-delà d'eux-mêmes, en eux-mêmes » (Arnaud \& Garrigue, 1982, 3).

\section{Lecture du texte, lecture de l'image : le recentrage disciplinaire (1995-2008)}

23 La rénovation du collège en 1995 reste associée à l'introduction dans les programmes de la grammaire de texte et de la grammaire de discours (Biagioli, 2016, p.605). L'approche des textes devient beaucoup plus technique, laisse peu de place au ressenti, davantage à une recherche des intentions de l'auteur outillée par l'analyse du discours. En même temps, la modélisation des types discursifs pourvoit la lecture d'instruments d'évaluation rapidement détournés en guides de production pour l'écriture (notamment le schéma narratif). Les apprentissages littéraires sont structurés autour des genres qui articulent étude de la langue et étude des textes. Une nouvelle forme d'organisation pédagogique apparait : la séquence. Elle décloisonne les sous-disciplines en les réunissant autour d'un unique objectif, le développement de la lecture littéraire. La production écrite devient à la fois plus réflexive, en termes de programmation et d'évaluation, et moins créative. Elle est essentiellement cantonnée à l'écriture hypertextuelle pour l'écriture longue et aux jeux poétiques pour l'écriture courte.

Quelle place reste-t-il pour l'intermédialité et l'interartialité ? Elles vont être à la fois appauvries et généralisées par la mise en place d'un parallélisme strict entre lecture de l'image et lecture de texte, parallélisme qui va accompagner l'apprentissage progressif des types discursifs (narration en $6^{\mathrm{e}}$, dialogue et description au cycle central, argumentation en $3^{\mathrm{e}}$ ) :

certaines images peuvent avoir fonction de narration. Les représentations imagées des évolutions de la chenille au papillon, du têtard à la grenouille ou du singe à l' 
homo sapiens, peuvent être lues ans ce sens. C'est également le cas de la bande dessinée $[. .$.$] ;$

certaines images peuvent avoir fonction de description: par exemple la représentation d'un navire dans un document à visée historique [.. .]. On associera l'approche de telles images à la sensibilisation à la description et aux lectures documentaires ;

certaines images, enfin, peuvent avoir un rôle de "preuves ", d'argument, comme, par exemple des photos de presse, les reproductions de documents authentiques [...]. On les abordera comme sensibilisation à l'argumentation. (Direction de l'enseignement scolaire, 1999, p. 38-39)

Seule la lecture des manuels - nous avons choisi le Texto collège $5^{e}$ (Besson, BastardGallet \& Raymond, 2001), très apprécié en son temps -, révèle ce que dissimule la clarté de cette progression: les degrés et les types différents de la sujétion de l'image au texte. Elle étaye les apprentissages de la langue et du discours, et lorsqu'elle est étudiée pour elle-même, c'est dans la même perspective que celle de la lecture méthodique. L'élève est invité à retrouver les structures qui ont guidé la production, en traçant les lignes de fuite du carrelage de la Vierge d'Autun de Van Eyck (ibid., p. 108), ou la charpente (diagonales et médianes) d'une illustration de F. Roca pour L'île au trésor de R. L. Stevenson. La différence des supports révèle le but de l'exercice : faire percevoir les structures communes à tous les genres iconiques. Cette démarche est complétée par des exercices de différenciation, comme la comparaison de trois images de lions : une photo de reportage animalier, un dessin de l'illustrateur du manuel montrant un lion de fable avachi, un bandeau sur l'œil, et une esquisse à l'encre de Rembrandt.

La lecture experte, de l'image comme du texte, s'arrête au seuil de la pratique créative. L'unique exemple de pratique interartiale auctoriale: un croquis de voyage (ibid., p. 200), n'est pas mis en rapport avec le texte des carnets de voyage dont il est extrait, mais plutôt avec la nature du texte : authentique versus fictionnel. On insiste sur son rôle mémoriel ("grâce à ce document, voyons-nous ce que l'auteur a vu à ce momentlà? Dites pourquoi cette page est un témoignage sur un lieu et un moment particuliers ? Pourquoi l'auteur a pris le temps de réaliser ce dessin?») sans égard pour son fonctionnement esthétique. La seule suggestion de projet créatif, qui a l'avantage de sortir du duo texte-image: "Voici à titre d'exemple un ballet pour te donner des idées. Tu peux aussi créer le tien » (ibid., p. 257) est donnée sans mode d'emploi, et dans un contexte tellement contraint (le ballet n'est là que pour étudier le rôle des éléments descriptifs dans l'identification des personnages au théâtre) que ni la spécificité du ballet, résumé à partir de tableaux statiques, ni celle de sa composition n'apparaissent.

Le parallèle constant entre texte-image met en place une pratique intermédiale resserrée, mais qui, à cause de l'implicitation ou de l'absence de contexte artistique, débouche rarement sur une interartialité resserrée. Cette dernière est davantage présente dans la transposition cinématographique du roman ou de la nouvelle, étudiée en $4^{e}$ et $3^{e}$. Elle donne lieu à quelques mises en pratique sans que l'on envisage cependant la réciproque de la transposition cinématographique : la romancisation du scénario filmique.

L'image restera durablement asservie au texte dans les représentations des enseignants. Pour certains, cette dépendance est un rempart contre sa prolifération incontrôlée :

si l'image est évincée des savoirs, c'est en grande partie à cause de son foisonnement: on a peur de se laisser entrainer hors des sentiers battus, on a scrupule à enseigner ce pour quoi on n'a pas reçu de formation, on a peur de la 
contestation, polie ou vigoureuse que suscitera tout à coup la polysémie de l'image.

(Serre-Floersheim, 2001)

29 leur propre interartialité, diversifiant les illustrations internes avec des pictogrammes qui réfèrent directement aux objets, et des dessins expressifs ou parodiques chargés de (télé)guider les réponses des élèves lorsqu'ils doivent identifier les registres. discipline sur elle-même et une tendance expansionniste. L'iconographie s'appauvrit en références artistiques mais importe des images, voire des séquences didactiques d'autres disciplines : l'histoire (documents, schémas, cartes) les arts visuels (leçons de lecture de la peinture, de la BD, de l'affiche de cinéma), les sciences de vivant (planche botanique). Et l'iconographie interne du manuel se développe. Revers du tout discursif, le tout iconique brouille le dialogue de la littérature et des arts.

\section{Histoire des arts : le français au défi de l'interdidactique (2008-2015)}

31 La réforme de 2008 introduit l'histoire des arts comme une matière interdidactique ${ }^{3}$ que chaque discipline est invitée à nourrir de ses apports. On peut la considérer comme une tentative de rééquilibrage du socle commun qui dépossède le français de ce qu'il avait commencé à entreprendre: la fédération de plusieurs disciplines autour des apprentissages langagiers.

L'introduction de l'histoire des arts participe d'une certaine façon à la déstabilisation de la représentation que nous avons de notre propre discipline, mais elle nous pousse en même temps à en préciser les contours. La nature étrange de cette étrange notion place les enseignants de français dans une position étrange. D'abord en retrait des textes officiels, ne figurant pas parmi les professeurs désignés pour l'évaluation, ils prennent désormais une place de plus en plus importante dans cette formation. (Baldner \& Barbaza, 2013, p. 5)

Dans leur présentation du numéro du Français Aujourd'hui consacré à la réception de la réforme, J.-M.Baldner et A.Barbaza, pointent trois difficultés: l'organisation didactique : «comment l'intégrer [l'histoire des arts] à sa discipline sans en faire un enseignement supplémentaire et dans une concordance didactique?» (ibid., p. 3), la formation des enseignants : «La transdisciplinarité qu'impliquent les textes peut-elle se faire sans une certaine universalité des enseignants?» (ibid.), et l'articulation de l'histoire des arts avec « la pratique artistique, domaine des professeurs d'arts visuels et appliqués, de musique et d'éducation physique » (ibid., p. 4). Cinq ans après la mise en place de la réforme, les enseignants de français ne sentent donc pas, ou pas encore, ou plus, autorisés à assumer l'enseignement d'une pratique artistique, fût-ce celle de la littérature, et se situent du côté des praticiens du discours sur l'art, plutôt que des praticiens de l'art. Ces différents problèmes soulevés confirment à la fois le statut épistémique et didactique incontournable de l'interartialité comme cadre d'articulation des savoirs scolaires entre eux et avec les pratiques sociales de référence, et la complexité de l'entreprise dont nous allons prendre une rapide mesure en parcourant quelques manuels.

Les programmes du français au collège de 2008 se signalent par l'abandon de « la lecture de l'image " au profit de " l'étude de l'image », un retour aux siècles littéraires qui facilite la mise en cohérence des programmes d'histoire et de français, 
l'introduction d'une dimension universaliste : « le regard sur le monde, sur les autres et sur soi à différentes époques en relation avec l'histoire de l'art ", et une insistance sur «la pratique constante, variée et progressive de l'écriture ». Les travaux d'écriture comportent des "écrits à partir de supports divers permettant de développer des qualités d'imagination (images, objets, documents audiovisuels), notamment en relation avec les œuvres étudiées le cadre de l'histoire des arts». Pour le français, l'ancrage suggéré avec l'histoire des arts est la thématique "Arts, États et pouvoirs ", qui permet de corréler périodes historiques, et domaines autour des œuvres étudiées. Une proposition que les manuels sont loin d'appliquer de façon rigide, réinjectant la progression historique dans des entrées qui restent liées aux objectifs majeurs de la discipline. Les titres de séquences de Français $5^{e}$ (Lagache et al., 2010) empruntent soit ceux des œuvres intégrales: Le Lion de Joseph Kessel, Le livre des merveilles de Marco Polo, soit des noms de genre: Le Roman d'aventures, de mythe littéraire: Robinson de Daniel Defoe, à Michel Tournier ou de forme littéraire: Jongleries verbales $d u X^{e} a u X^{e}$ siècle.

Cette transition éclairée semble avoir été propice à l'apparition de dispositifs de formation à l'interartialité. Côté réception, on met en valeur l'interartialité du corpus patrimonial. Le rondel lié à la musique et à la danse vient équilibrer l'indétrônable calligramme (ibid., p. 245). V. Hugo est enfin reconnu comme dessinateur. Un montage le montre entouré de ses dessins les plus connus (Briat et al., 2011, p. 135). On met son poème D'après Albert Dürer, en rapport avec l'hypotexte iconique (Lagache et al., p. 259). On analyse des représentations picturales de communautés artistiques et littéraires (Un coin de table de Fantin-Latour [Briat et al., 2011, p.17], Le salon de Mme Geoffrin de Lemonnier [ibid., p. 18]). On étudie la représentation de la pratique musicale dans la peinture (ibid., p. 252). On rappelle que Jean Renoir n'est pas seulement l'auteur de La Bête humaine, et de Nana, mais aussi de La grande illusion et de La règle du jeu et qu'il est le fils du " peintre impressionniste Auguste Renoir » (ibid., p. 44-45).

Deux nouveautés : l'insert documentaire sur une technique artistique (ex: Les procédés photographiques [ibid., p. 81]); et le groupement thématique (ex : L'ile dans l'art moderne, qui regroupe peinture, land art et architecture [Lagache et al., p. 128-129]), transposition aux arts du groupement de textes.

La production intermédiale reste le parent pauvre. Assez bizarrement l'exploitation du carnet du voyage se limite à une fiction métatextuelle: «En une dizaine de lignes, imaginez et rédigez les commentaires que vous-même pourriez faire dans votre carnet de voyage après avoir découvert une "merveille" dans un pays lointain. Consignes : comme Marco Polo, adressez-vous au lecteur et décrivez avec précision et admiration la "merveille". Vous veillerez à utiliser au moins un superlatif » (ibid., p. 110), alors qu'on aurait pu inciter l'élève à faire un vrai périple et à écrire à partir de ses propres croquis. Des démarches créatives oubliées resurgissent. "Créer un animal symbolique: Racontez en quelques phrases la naissance de cet animal et trouvez-lui un nom. Illustrez votre texte par un dessin ou un collage» (Potelet, 2014, p. 63), retrouve la dynamique interartiale de la co-construction du texte et de l'illustration, lorsque celleci est réalisée soit par l'écrivain, soit en collaboration avec l'illustrateur. Le support iconique à référence indécidable (un paysage de Turner) pour « composer un poème en vers libres célébrant un paysage réel ou imaginaire» (Briat et al., 2011, p. 223), se souvient du texte libre sur embrayeur iconique. Le recours à la photo comme lanceur d'écriture n'est pas nouveau, mais reflète la métamorphose du média en demandant de 
créer un haï-ku à partir d'une photo prise avec un smartphone, instrument intermédial familier et relevant lui aussi d'une culture de l'instant.

L'instauration du Parcours d'éducation artistique et culturelle de l'élève (PÉAC), de 2013 à 2015 , répond à certaines des interrogations soulevées par la mise en place de l'histoire des arts en définissant le cadre de l'éducation artistique et culturelle :

Le parcours d'éducation artistique et culturelle est l'ensemble des connaissances acquises par l'élève, des pratiques expérimentées et des rencontres faites dans les domaines des arts et du patrimoine, que ce soit dans le cadre des enseignements, de projets spécifiques, d'actions éducatives, dans une complémentarité entre les temps scolaire, périscolaire et extrascolaire. (Arrêté du 7 juin 2015)

Celle du système d'évaluation par compétences généralise le principe de la prise en charge commune d'un objet d'enseignement, inauguré par l'histoire des arts, à tous les objets d'enseignement. Dans les blocs de compétences, la communication interartiale est à la fois partout et (presque) nulle part. Partout, parce que sa base : l'intermédialité, devient la règle. La «lecture autonome de textes variés, d'images et de documents composites, sur différents supports » (MEN, 2016, p. 2), concerne toutes les disciplines. Nulle part, parce que les conditions d'une communication interartiale effective sont rarement réunies. Le projet personnel créatif est décliné en parallèle en musique, dans les arts plastiques et en français (ibid., p. 12). Les disciplines ne se rejoignent pas. C'est l'élève qui, sur son carnet de bord, tient le registre compétences acquises (ibid., p. 13). Il faut atteindre la dernière page pour trouver une formulation qui corresponde à l'une de ses composantes de la compétence interartiale: "Pratiquer divers langages artistiques en lien avec la connaissance des œuvres et les processus de création » (ibid., p. 24).

Dans les manuels de 2016, la majorité des activités concernent la lecture, une lecture impactée par l'interartialité à travers deux genres scolaires: le groupement thématique, et la lecture comparative. Introduit en 2008, le groupement thématique autour de mythes, symboles, et autres universels-singuliers interculturels, se généralise et constitue une véritable initiation à la littérature comparée. La lecture comparative, comme celle de Pour faire le portrait d'un oiseau de Prévert et de La clairvoyance de Magritte qui montre un peintre peignant un oiseau en regardant un œuf (Beltrando, 2016, p. 180-181), contrairement à la lecture traditionnelle du texte-image, ne s'arrête pas aux ressemblances thématiques ou stylistiques mais s'attache à expliciter les démarches poétiques de chaque œuvre.

41 Cependant une consigne d'écriture argumentative atteste la volonté de transposer à un niveau interartial une problématique apparue en littérature: «Comment imaginezvous qu'un cinéaste, un musicien ou un peintre puisse, comme Rimbaud, "écrire des silences" ? Illustrez vos arguments d'exemples tirés de votre culturel personnelle» (Bertagna \& Carrier, 2016a, p.311). Un concours de pocket films poétiques sur l'électricité réalisés avec l'application caméra vidéo d'un smartphone (Bertagna \& Carrier, 2016b, p.157), articule approche poétique et approche scientifique. Le smartphone se retrouve dans une présentation de l'art selfie (ibid., p. 37), autour d'une photo d'un portrait de femme de Jordaens prise avec la main actionnant le smartphone au premier plan, ce qui déconstruit l'effet de la reproduction et permet d'engager la réflexion sur une pratique de consommation culturelle naturalisée par l'habitude.

L'objet 3D est réintroduit comme objectif de production dans un atelier de théâtre d'objet, théâtre de marionnettes fabriquées à partir d'objets courants (Randanne, 2016, 
p. 156), avec des conseils sur le choix des matériaux (en rapport avec les thèmes de l'œuvre, la réalisation, et la manipulation des marionnettes), et deux photogrammes d'une représentation de l'Avare, dont un gros plan sur les marionnettes. La consigne : « vous décidez de jouer l'une des scènes de cette séquence en manipulant des objets qui seront les personnages » (ibid.), s'en remet aux compétences des élèves sans évoquer un quelconque encadrement. Elle exploite une tradition interartiale qui est aussi une modalité d'apprentissage connue des professionnels du théâtre pour faciliter, grâce à la médiation des marionnettes, l'immersion dans la fiction dramatique.

Ce survol du dialogue de la littérature et des arts dans les instructions et les manuels depuis 40 ans laisse l'impression d'une avancée spiralaire qui revient et repart sans arrêt depuis sa double base, le texte et l'image, pour intégrer peu à peu les changements culturels et technologiques, et s'accélère brusquement lorsque l'intégration des nouvelles technologies, de l'interculturalité et de la fréquentation des œuvres démocratise l'offre de formation à l'interartialité. Toutefois cette offre est beaucoup plus axée sur la réception que sur la production, ce qui en limite la portée. Mais alors que l'image et l'objet sont plus faciles à déchiffrer et deviennent plus faciles à produire que le texte, développer la pratique des arts non verbaux dans les activités interartiales ne risque-t-il pas de compromettre le développement des apprentissages langagiers au lieu de les étayer? C'est cette interrogation qui a inspiré le recueil des données que nous allons maintenant analyser.

\section{L'interartialité dans les pratiques}

Nous avons demandé à un collègue enseignant de français au collège Leï Garrus à Brignole, Christophe Raoux, associé aux recherches de notre laboratoire ${ }^{4}$ depuis 2007, si lui et ses collègues avaient pratiqué dans leurs classes des activités d'écriture interartiale depuis la rentrée 2016. Avec lui, nous avons sélectionné deux de ces activités. L'une à partir de l'image, parce qu'elle avait la particularité d'être effectuée dans le cadre d'une relation suivie avec un écrivain, et que c'était la première œuvre interartiale de cet écrivain qui constituait son point de départ. L'autre, compte-rendu d'une lecture cursive via une production d'objet, nous paraissait de nature à vérifier le fonctionnement d'une interartialité complète et non restreinte à l'image. Les réunir permettait de confronter les effets de la médiation interartiale sur l'écriture dans les deux sens art-texte et texte-art. Un à trois mois se sont écoulés entre la réalisation des activités et l'enquête, ce qui a facilité le retour réflexif sur les pratiques. Les questionnaires ont été revus et retouchés par notre collègue avant d'être distribués. Cette recherche est empirique. Si certains recoupements ont permis d'esquisser des hypothèses, c'est uniquement à titre de pistes qui ne pourront être vérifiées que par des dispositifs plus vastes et/ou spécialisés.

\section{Écrire à partir d'une image}

L'interartialité est largement attestée dans les pratiques sociales de référence des écrivains et des artistes. Cependant, nous l'avons vu, elle a longtemps été tenue pour marginale dans l'enseignement de la littérature, ce qui pose le problème de l'utilisation des pratiques sociales de référence dans les dispositifs didactiques. Tout comme la conception de l'ingénierie didactique étudiée, nos questionnaires ont tenu compte des 
trois facteurs soulignés par C. Cohen-Azria (2010, p. 179) : « les pratiques renvoient aux activités "réelles" d'un espace social identifié qui peuvent servir de référence pour la conception ou l'analyse d'activités scolaires ».

La classe enquêtée est une $6^{\mathrm{e}}$ de 26 élèves qui pratique l'écriture en atelier une fois par quinzaine pour les textes courts, toutes les trois semaines pour les textes longs. La visite de l'écrivain a pris place juste après la lecture en œuvre intégrale du premier tome de sa saga. Ce n'est qu'ensuite que l'enseignant a fait écrire un texte à partir de la première de couverture du second tome, en effaçant le titre pour éviter l'effet de continuation. Les élèves savaient que l'écrivain était l'auteur de l'illustration. L'activité a duré 55 minutes Ils avaient la possibilité de travailler en groupe. De ce fait, les textes les plus aboutis ont été les textes collectifs mais tous ont pu répondre au questionnaire qui comportait trois parties relatives à la production, l'évaluation, et la conscientisation de l'interartialité.

Nous avons commencé par nous enquérir du degré de familiarité des élèves avec la tâche. Onze n'avaient jamais écrit à partir d'une image, 4 ne s'en souvenaient pas. Six se souvenaient du genre du texte (description, fiction autobiographique, suite) ; $2 \mathrm{du}$ genre de l'image (une première de couverture); aucun des deux à la fois. Parmi ceux qui se rappelaient avoir travaillé sur une première de couverture : 14 , un seul a pu citer le titre de l'œuvre : Le Petit Nicolas. Un autre se souvenait vaguement que : «ça parlait d'une monstre ", un autre a décrit la couverture: "c'était un village dans une forêt entourée de flammes non loin des montagnes ». La première de couverture est donc assez faiblement corrélée au titre, quoique comprise dans son fonctionnement de seuil : à la fois équivalent de, et introductrice au texte.

Deux questions portaient sur la spécificité du dispositif proposé : « selon vous, qu'est-ce qui peut changer quand l'écrivain illustre lui-même son œuvre au lieu que ce soit une personne différente?». L'autre demandait si l'absence de titre: facilitait l'invention, obligeait à se souvenir du premier tome pour deviner la suite, à accorder plus d'attention à l'image, ou donnait l'idée d'écrire autre chose qu'un récit. La première a désarçonné 11 élèves, et obtenu 14 réponses. Toutes valorisent le procédé, insistant sur la connaissance que l'auteur a de son projet, son imagination, sa capacité à émouvoir : « il peut donner plus de sentiment à son illustration ", à être ému : «il ressent quelque chose d'autre et est fier de lui », comme si la première de couverture était un regard satisfait que le créateur - tel le dieu de la Bible - jette sur son œuvre avant de la livrer au public. Parmi les conséquences de la suppression du titre, la possibilité d'imaginer une autre histoire a obtenu 18 suffrages, la centration de l'attention sur l'image 9 , contre 3 pour la suite non narrative.

Les questions 5 à 11 étaient consacrées au passage de l'image à la conception du texte. La 5 recentrait la mémoire sur l'image en demandant de la décrire. Les réponses sont descriptives (centrées sur le paysage : un lac avec un pont dont on ne voit pas le bout, des montagnes à l'arrière-plan), narratives (centrées sur le personnage : un joueur de flute) - la majorité -, ou émotionnelles : "pont sans fin ", "paysage immense ». Une réponse indique simplement "le désespoir». Une autre se souvient d'icebergs qui n'existent pas sur l'image. Certaines omettent le soleil, d'autres les montagnes. Ces résultats renvoient au concept de spectature (Lacelle, 2009) qui prend en compte, parallèlement à la lecture de l'écrit, des caractéristiques sémiotiques et subjectives propres à la réception de l'image: globale, sélective, associative, subjective. Nous demandions ensuite aux élèves s'ils avaient eu envie de: 1) rentrer dans l'image, 
comme un personnage, 2) raconter ce qui se passait, comme un narrateur, 3) s'intéresser à la scène mais seulement comme un spectateur, 4) modifier la scène. On pouvait cocher plusieurs réponses. L'immersion fictionnelle centrée sur le personnage a obtenu 11 voix, l'immersion fictionnelle décentrée 9, la reprise de l'image par la narration 7 , et la transformation de la scène 6 . Les postures les plus représentées sont donc celles soumises à l'attractivité de l'image. On note toutefois que les deux postures immersives s'opposent par le degré d'immersion. Or elles ont été couplées quatre fois, ce qui indique qu'elles peuvent faire l'objet d'une alternance de centration et de décentrement caractéristique du passage du statut de spectateur au statut de narrateur. D'ailleurs celui-ci a été couplé trois fois avec l'immersion identificatoire et deux fois avec l'immersion distanciée. En revanche les 6 personnes qui ont eu envie de modifier la scène n'ont coché que cette case. Ayant refusé d'emblée de rentrer dans l'image, elles n'ont pas eu à en sortir. Le genre des textes produits (question 7) fait ressortir le récit à une écrasante majorité. La poésie n'obtient que 4 voix. Les réponses concernant le registre (question 8) montrent que les élèves savent le distinguer du genre (une seule non-réponse). Le registre poétique est coché par ceux qui avaient choisi le genre poésie, mais aussi par un élève qui avait choisi le roman (l'image était assez onirique). Les registres aventure (9 voix), fantastique (7), énigme (1) complètent l'identification générique.

Les questions 9 et 10 demandaient de détailler la transposition au niveau du contenu, en identifiant les éléments de l'image qui avaient été gardés et ceux qui avaient été supprimés. Alors que la 10 avait été posée dans la simple intention de vérifier les réponses à la 9 , le taux d'échec est passé de 8 à 12 personnes. Plusieurs facteurs sont à prendre en compte. D'abord la mémoire : les non-réponses ou les aveux d'impuissance après effort («j'sais pas, désolé ») sont le fait de personnes qui avaient déjà eu du mal à décrire l'image. Ensuite la nature des opérations. La seconde est plus complexe que la première : se rappeler ce qu'on a gardé demande une addition, se rappeler ce qu'on a supprimé suppose deux opérations, l'addition récapitulative puis la soustraction. Par contraste, les réponses globales (« rien, tout, un peut de tout »), traduisent non pas une absence de précision dans la transposition, mais un ressenti privilégiant la perception ensembliste des détails, ce qui est normal à cette étape préliminaire de la transposition où il fallait tirer de l'image l'« idée » du nouveau scénario.

51 La question 11 portait sur l'énonciation. Conformément au programme, nous avions limité le choix à narrateur intérieur ou extérieur, sachant que les manuels les identifient aux pronoms de $1^{\text {re }}$ et $3^{\mathrm{e}}$ personne pour faciliter leur repérage $\mathrm{e}^{5}$. Nous avons obtenu 9 réponses pour le narrateur extérieur dont 4 justifiées et 11 pour le narrateur intérieur dont 5 justifiées. Une réponse précisait : « les deux » : ce qui est évidemment possible dans un récit romanesque un peu étoffé, mais qui s'explique surtout par l'assimilation du régime narratif au pronom (le pronom de $1^{\text {re }}$ personne peut apparaitre dans un dialogue sans désigner pour autant le narrateur). Une autre: "aucune des deux, on a fait de la poésie ", révèle une confusion entre genre et discours liée à l'association systématique du registre narratif aux genres narratifs. Les justifications adoptent le point de vue de l'auteur : «pour s'y croire ", " pour se mettre dans la peau du personnage », « parce que j'aime bien » (narrateur intérieur), « je n'avais pas envie de faire partie de l'histoire » (narrateur extérieur) ; ou celui du lecteur : « parce que j'ai trouvé que ça faisait mieux », " car je trouve que c'est plus facile à lire » (narrateur intérieur). Deux justifications du narrateur extérieur : "Car c'est moi qui raconte » et 
« car je suis le narrateur et le personnage principal et aussi parce que je raconte ce qui se passe » montrent une difficulté à distinguer auteur et narrateur.

Pour documenter la réalisation du texte, nous demandions un récit : "Comment avezvous fait pour écrire ce texte » (12), et un ressenti : «Qu'est-ce qui vous a paru le plus dur ? le plus facile?» (13). Les réponses (19) se répartissent entre l'écriture spontanée : "j'ai écrit d'un coup ", «j'ai écrit tout de suite », «j'ai écrit directement sur ma feuille sans faire de brouillon »; et l'écriture contrôlée : «j'ai cherché dans ma tête, j'ai écrit », " on s'est réuni tous ensemble, on s'est donné des idées, puis après on a commencé à écrire ». Elles confirment la place prépondérante de la recherche des idées dans la planification (Fayol, 1996, p. 21) et la séparation entre l'étape de récupération des idées et celle de leur organisation (ibid., p. 22-24). Celle-ci est facilitée par la chrono-logique du récit, ce qui explique que l'expression des choix génériques se soit faite selon une ligne de faille récit/ poésie, la poésie autorisant une organisation plus floue et répétitive.

53 Autre confirmation, celle d'une résistance au contrôle scriptural, qui se manifeste par le rejet du brouillon mais peut s'expliquer aussi bien par un défaut de conscientisation des étapes («je ne sais plus les étapes») que par l'envie de ne pas ralentir les idées lorsqu'elles viennent. On remarque aussi que le contrôle parait plus naturel dans le travail de groupe. Quelques réponses sont axées sur la dimension interartiale : «j'ai juste regardé l'image jusqu'à avoir une idée ", «j'ai regardé l'image et j'ai marqué tout de suite », « on a commencé au bas de l'image et on est monté ».

Dans l'évaluation des difficultés et des facilités, on retrouve la globalisation: «rien n'était facile ", "tout était difficile ", "rien ne m'a paru dur mais plus facile grâce à l'image » et la difficulté de trouver les idées, contrebalancée à ce stade par les souvenirs des difficultés d'écriture : "le plus dur c'est de raconter l'histoire ", " décrire l'image ", «inventer les noms ». Une réponse remonte au tout début du processus : «le plus dur c'est d'entrer dans l'image ». Deux insistent sur la rédaction : « le plus dur c'est d'écrire la fin, le plus facile c'est de commencer le texte ", « le plus facile c'est le début et la fin, le plus dur c'est le milieu ", révélant des degrés différents de la planification narrative. L'image peut donc aider à trouver les idées, mais à condition qu'on puisse s'y investir, et durant la rédaction, offre un étayage descriptif mais peut également décourager parce qu'elle est plus difficile à décrire qu'à lire.

Les questions suivantes portaient sur l'évaluation du travail par l'enseignant (14), les pairs (15), la réaction des scripteurs à l'une et à l'autre $(16,17)$, et sur leur propre évaluation des textes de leurs camarades (18). Tous les élèves n'ayant pas rendu de texte, il y a eu 9 non réponses. Nous avions donné 4 critères : contenu, forme, rapport image/texte, singularité du texte (" ce que votre texte avait de différent des autres »). Le plus cité a été le rapport image/texte, 10 fois pour le professeur 6 pour les pairs. La forme est prise en compte davantage par le professeur que les élèves, et vice-versa pour le contenu, la singularité du texte un peu plus par les élèves que par les professeurs. La nature interartiale de la tâche a donc été conscientisée. Les élèves sont en général d'accord avec les remarques de l'enseignant et de leurs camarades, un peu plus toutefois avec l'enseignant qu'avec leurs pairs. En revanche, ils deviennent subitement amnésiques quand on leur demande "vous-mêmes, avez-vous réagi aux textes de vos camarades si oui, sur quoi avez-vous insisté dans vos remarques». Seulement deux réponses développées, mais sans rapport avec les critères : L'une plutôt acerbe : «oui qu'il y avait pratiquement la même chose », l'autre diplomate : « oui j'ai réagi à certains 
textes, mais je n'ai pas forcément insisté ». Les deux signalent la difficulté à engager un groupe dans une démarche d'évaluation croisée lorsque les personnes s'identifient à leurs productions.

Les questions 20 et 21 concernaient la mise en relation de la pratique scolaire avec la pratique sociale de référence. La question 20 : «Aimeriez-vous que l'auteur du livre lise votre texte ?» (rappelons que les élèves le connaissaient, et avaient beaucoup apprécié l'échange qu'ils avaient eu avec lui) a obtenu 13 réponses positives, et 10 négatives. On souhaite avoir l'avis de l'auteur par curiosité : «pour savoir sa pensée », ou pour bénéficier d'un avis autorisé : «j'aimerais bien pour qu'il me corrige et qu'il me dise mon travail s'il est bien ou pas", "pour savoir si ça commence bien». Certains se posent en collaborateurs: "je veux qu'il voit mon imaginassions", «ça pourrait lui donner d'autre idée ». Les refus trahissent une image du soi scripteur fragile, redoutant l'évaluation: " parce qu'il pourrait nous juger ", prompt à l'autodénigrement : "parce que je n'ai pas inventé un beau texte », « car c'est nul », se repliant sur l'école et sur la vie privée : «je préfère garder le texte pour moi », «je préfère le garder pour moi et la classe ». Mais on trouve aussi une appréciation lucide de l'écart entre pratique scolaire et pratique sociale de référence : « car ce n'est pas à la hauteur ».

Avec la question 20 : «Aimeriez-vous comparer votre texte et ceux de vos camarades au livre une fois que vous l'auriez lu? », nous souhaitions vérifier si la relation amicale et professionnelle avec l'auteur permettait de surmonter l'effet dévalorisant du dévoilement de la fin authentique à l'issue d'une activité de continuation. Il y a eu 13 réponses négatives et 11 positives. Les raisons du refus confirment celles données à la question précédente : «car je sais que le mien est nul », « car ils vont se moquer », «je n'aime pas être comparé ", " parce que ça ne sert à rien, on n'est pas en compétition », " pas envie, je préfère le garder pour moi ». Une vision de l'écriture très personnelle qu'un phasage lourd de la pratique scolaire avec la pratique sociale de référence, du type concours de nouvelles ou de poésie, pourrait facilement déstabiliser. Les réponses positives considèrent le texte authentique plutôt comme embrayeur d'imagination que comme source. La comparaison les intéresse moins pour juger que pour découvrir les solutions : "pour voir quelle idée ont eu les autres", "pour voir si avec certaines personnes on a eu les mêmes idées ", et les mutualiser : "ça donne des idées aux autres camarades », «j'aimerais bien pour réunir nos idées ».

Les dernières questions portaient sur les deux conditions fondamentales de la didactisation de l'interartialité : la prise en charge interdidactique et la réciprocité des interactions artistiques. La question 21 «A quelle(s) autre matière(s) que le français vous fait pense cet exercice? ? a déclenché la perplexité ( 5 «je sais pas »), le rejet ( 7 « aucun », 4 « que français », ou « juste français »). La réponse : «ça me fait penser à la littérature » renvoie à la notion de configuration disciplinaire (Reuter, 2010, p. 88), qui désigne les variations de l'organisation des enseignements d'une discipline selon les niveaux et les sections. En $6^{\mathrm{e}}$ la littérature peut très bien apparaitre comme une discipline nouvelle. Quatre références extérieures à la discipline ont été proposées : les arts plastiques (réponse attendue puisque c'était l'autre pratique créative convoquée par l'activité), mais aussi l'histoire, les SVT et la physique-chimie. M. Fayol (1996, p. 23) rappelle que «non seulement les produits sont de meilleure qualité quand on a des connaissances préalables [...] mais aussi que le cout cognitif de la production est bien inférieur ». Les disciplines non concernées par la forme d'une œuvre littéraire peuvent très bien l'être par le contenu. L'histoire, seule discipline coordonnée avec le français 
dans les programmes, sert à contextualiser les œuvres, ce qui relève de la lecture littéraire. L'écriture littéraire, elle, est susceptible de mobiliser n'importe quel type de savoir, en fonction de l'univers représenté.

La question 22: "Aimeriez-vous faire une image pour illustrer un texte? " a obtenu 18 réponses positives et 7 négatives : la réversibilité des opérations interartiales semble admise. Restait à dire comment on s'y prendrait (question 23). Sur les dix propositions récoltées, certaines prennent pour point de départ l'image mentale : "prendre l'image de son imagination ", "prendre des idées un peu bizarres dans sa tête», d'autres le texte : "avoir déjà l'idée du texte ", «inventer d'abord le texte dans notre tête puis dessiner », d'autres l'image réelle : « il faudrait faire une image (dessiner) ». On trouve aussi deux propositions d'ingénierie interartaile : « faire une image en arts plastiques et ensuite inventer un texte en français » et " une classe dessine un dessin et une autre invente une histoire grâce aux images de l'autre classe ».

Pour compléter et éclairer la description des pratiques des élèves, nous avions demandé à l'enseignant et à l'écrivain de répondre chacun à un questionnaire. Nous résumons ici les points de leurs réponses qui nous ont semblé le mieux illustrer l'actualisation de la pratique sociale de référence, celle de l'interartialité et les conséquences sur les apprentissages

61 L'écrivain est un neuro-psychologue de 29 ans - ce qui confirme ce que l'on sait de «la double vie des écrivains" (Lahire, 2006). Ce n'est pas un débutant (deux ouvrages publiés, un troisième en projet). Il ne se définit pas spécialement comme un écrivain pour la jeunesse. Comme tout écrivain, il a une filiation littéraire. Ses auteurs favoris sont P. Bottero qui lui a donné l'envie d'écrire, P. Pullman, J. K. Rowling, A. Damasio, P. Pevel, à propos desquels il préfère parler de « littérature de l'imaginaire " plutôt que de fantasy. Les thèmes qu'il aborde dans ses livres sont la peur, l'oppression, la tyrannie, mais aussi la liberté et la résilience, la famille, la force de l'amour et de l'amitié, et « le rôle que les émotions jouent dans notre vie ».

62 L'enseignant a 39 ans, et 16 ans d'ancienneté dans la carrière dont 13 dans l'établissement. Étudiant il a écrit des poésies et des nouvelles. Actuellement il a un projet d'écriture à quatre mains avec un ami qui écrit depuis longtemps. Cette familiarité avec la pratique sociale de référence a rejailli sur son métier. Il pratique l'atelier d'écriture dans ses classes, et fait améliorer les textes si c'est un travail sur le long terme "création d'un conte long par exemple, qui sera relié et donné aux parents ». Il invite des écrivains en se fondant sur quatre critères : il les connait, il apprécie leurs œuvres, les élèves aussi, et il sait que l'écrivain a un bon contact avec les jeunes. L'un d'eux était P. Bottero.

63 L'écrivain reconnait être inspiré, de façon plus ou moins consciente, par d'autres arts que la littérature comme le cinéma et la musique. Il ne se considère pas comme un illustrateur mais a décidé d'illustrer la couverture de son deuxième livre parce qu'il prend des cours de dessin et que l'une de ses collègues de cours avait peint l'illustration de couverture de son premier livre qu'elle avait lu et apprécié : «j'ai trouvé cela parfaitement complémentaire et dans la continuité de mon travail d'écrivain : s'évader non pas par les mots, mais par le pinceau!». Pour lui, «l'auto-illustration reflète le ressenti de l'auteur sur son histoire", ce qui rejoint l'avis des élèves, tandis que "l'hétéro-illustration permet de mettre en lumière la vision qu'a un lecteur/ illustrateur de l'histoire, vision qui peut être très différente de la vision que l'auteur a 
de sa propre histoire ", un besoin d'accéder au lecteur réel que l'on retrouve dans les motifs de sa visite. rarement ( 3 ou 4 fois/an) les images comme embrayeurs d'écriture, à partir d'une première de couverture ou d'une illustration à l'aveugle, soit pour développer des compétences d'écriture fictionnelle, soit pour travailler un point de langue. Cet habitus professionnel correspond à ce que nous avons observé dans les manuels et aux réponses des élèves lorsqu'on les interroge sur leur connaissance de l'exercice. L'utilisation de l'image comme embrayeur d'écriture indifférencié tend à la désartialiser, d'où l'importance de la réartialisation qu'opère la rencontre de l'auteur juste après la lecture de son œuvre.

en classe de l'écrivain reposait sur une négociation interindividuelle et non sur un dispositif institutionnel. Ce que souhaitait l'auteur, c'est « avoir le retour de jeunes adolescents [...] qui ont l'âge du héros principal », un retour libre et franc: "si les extraits des livres ne leur ont pas plu, qu'ils n'hésitent pas à le dire ». Il pense que les élèves attendent de lui qu'il soit ouvert à la discussion, " quelqu'un qui parle avec leurs mots à eux, et non pas quelqu'un qui s'écoute parler ». Dans les objectifs proposés, il a coché « prolongement du métier d'écrivain ", «façon de connaitre ses lecteurs », et ignoré «métier différent », « collaboration entre deux métiers différents » et "retour aux sources des apprentissages scripturaux ». Il juge que les textes écrits à partir de son illustration doivent être discutés en commun et rester la propriété exclusive de leurs auteurs si ceux-ci le souhaitent, avis qui réunit les deux options opposées rencontrées dans la classe. Il ne se prononce pas sur l'opportunité de leur évaluation.

L'enseignant a invité l'auteur pour "parler de son œuvre et du métier d'écrivain ", "des phases de l'écriture dans la création d'une œuvre littéraire » et non "pour faire écrire les élèves ». Pendant la rencontre, il préfère s'effacer et profiter de l'échange entre les lecteurs et l'auteur qu'il juge très intéressant. L'intervention de l'auteur est pour lui «un prolongement naturel des apprentissages", "une ouverture sur la pratique sociale de référence", "une façon pour les élèves de connaitre les professionnels de la littérature ", mais pas une situation d'intervention croisée qui lui permettrait de voir «si la présence de l'auteur transforme la façon de travailler des élèves ».

La rencontre est donc conçue comme une phase de différenciation qui établit un parallèle entre pratique sociale de référence et pratique scolaire. Le pivot de cette différenciation est le partage de l'expérience de la lecture de l'œuvre avec l'auteur. L'incidence sur les apprentissages n'est pas technique mais psychologique. Selon l'enseignant " quand [les élèves] voient, pour un travail d'écriture long, qu'ils passent par les mêmes étapes que des "vrais auteurs" (je les cite), ça les valorise et ça valorise aussi (voire même ça légitime à leurs yeux) le travail d'écriture qu'ils sont en train de faire ». La conséquence sur la pratique de l'interartialité est la recontextualisation de l'image dans le processus de création artistique.

\section{Fabriquer un objet à partir d'un texte}

L'objet 3D ne fait pas (encore) partie du contexte ordinaire du cours de français. La pratique interartiale que nous allons analyser maintenant peut être considérée comme innovante, autant que l'était la proposition de théâtre d'objet dans le Français $4^{e}$

Pratiques, 175-176 | 2017 
(Randanne, 2016). Son interartialité est à la fois robuste, parce que, comme dans l'exemple précédent, la partie littéraire consiste en la lecture d'un ouvrage explicitement désigné comme littéraire et qu'elle constitue un exemple de retour de l'art plastique sur le texte ; fragile, parce que la qualité de la réalisation plastique peut être sujette à caution, en raison de l'inexpérience des acteurs et qu'aucun représentant de la pratique sociale de référence n'y a pris part. Un autre facteur doit être pris en compte, c'est la nature métissée des objets qui devaient remplacer la fiche de lecture tout en intégrant certains de ses aspects (ex : bribes de ressentis de lecture inscrites sur des languettes en forme d'éclats de verre évoquant l'accident de voiture qui a causé le mutisme du personnage principal). Ce sont des objets parlants, à l'instar des vases et meubles parlants du verrier et ébéniste français, É. Gallé (1846-1904), qui marquetait sur ses œuvres non seulement sa signature et leur titre, mais aussi des citations de ses poésies favorites, des maximes ou des refrains de chansons. Cette pratique, celle de l'emblème, facilite l'entrée dans la communication esthétique parce qu'elle réfère au vécu culturel du récepteur, et procure à l'œuvre une forte valeur ajoutée, en termes de saturation signifiante et de densité émotionnelle continue mais à faible activation, paramètres définitionnels de l'attention esthétique selon J.-M. Schaeffer (2015, p. 55 et 125).

L'activité étudiée étant proposée en option et par seulement deux enseignantes, nous avons dû pour récolter un nombre suffisant de réponses mobiliser tous les élèves qui l'avaient réalisée : 8 élèves de $6^{\mathrm{e}}, 4 \mathrm{de} 4^{\mathrm{e}}, 9 \mathrm{de} 3^{\mathrm{e}}$, en tout 21 . Comme pour l'étude précédente, le questionnaire comportait trois parties relatives à la production, l'évaluation et la conscientisation de l'interartialité. Nous avons commencé par situer la pratique dans le temps. 19 élèves sur 21 ont répondu, 2 avec la date complète, 8 seulement le mois. À côté du temps civil, on trouve des mentions du temps scolaire: trimestre, tâche (« quand ma professeur de français nous a donné à lire notre deuxième livre de l'année»), et du temps privé: «c'était dans un week-end»). Quatre ont répondu à côté en dévoilant toute de suite leur production : «j'ai créé une boite avec des feuilles de bristol qui présentent le livre » $\left(3^{\mathrm{e}}\right)$, et même le titre et l'auteur du livre que nous ne demandions qu'aux questions suivantes, tant l'expérience les avait marqués. Tous les élèves ont su donner le titre du livre, performance sans doute facilitée par la restriction de la liste imposée au début de l'année. Sept avaient oublié l'auteur. L'auteur dont le nom a été le plus mémorisé est... P. Bottero.

70 Les questions 4 à 7 entraient plus avant dans la remémoration de la lecture. Tous les élèves ont su dire de quoi parlait le livre (question 4), de façon générique : "enquête policière ", synthétique: "d'un garçon handicapé qui voulait courir ", en tenant compte de l'énonciation: "d'une fille qui raconte sa vie. Son père est mort dans un accident de voiture et son frère est handicapé », en restituant le schéma narratif : «ce livre parlait d'un lycéen Ses amis sont morts, indirectement par sa faute. Le livre racontait sa vie dans un nouveau lycée, et comment, grâce à un nouvel ami, il arrive à aimer la vie de nouveau ». La longueur des résumés franchit un seuil entre la $6^{\mathrm{e}}$ et la $4^{\mathrm{e}}$, niveau à partir duquel on trouve des textes à effet d'annonce qui rappellent le style de la quatrième de couverture : " un virus a décimé toutes la population mondiale sauf les adolescents qui ont entre 14 et 18 ans. Des adolescents doivent se rendre à un étrange rendez-vous à Paris pour retourner dans le passé ».

71 La question 5 portait sur le nombre de pages et la 6 sur le temps de lecture. La longueur, adaptée au niveau, n'excède pas 150 pages pour les $6^{\mathrm{e}}$. En $3^{\mathrm{e}}$, les 300 à 
400 pages de chaque tome de la saga dystopique de J.-L. Blondel ne semblent pas avoir effrayé les élèves qui y retrouvent un univers qu'ils connaissent bien, celui du jeu vidéo, et une dramatisation ciblée sur leur tranche d'âge. Les écarts entre les lecteurs semblent se creuser d'un niveau à l'autre : deux jours à quelques semaines en $6^{\mathrm{e}}, 3 \mathrm{~h}$ à 2 semaines en $4^{\mathrm{e}} ; 1 \mathrm{~h}$ à 1 mois, et si l'on tient compte du rapport entre longueur et durée, de 150 pages en 1 mois à 340 pages en deux jours, en $3^{\mathrm{e}}$. Si une élève reconnait qu'elle lit " très doucement ", une autre précise qu'elle $a$ "lu en plusieurs fois » : on ne peut donc pas systématiquement assimiler longueur et difficulté de lecture.

question suivante demandait si la lecture était choisie ou imposée. Nous ne cherchions pas forcément la précision factuelle mais plutôt un ressenti, car nous connaissions le statut institutionnel paradoxal de la lecture cursive. Dans l'idéal, c'est un pré-requis de la lecture expliquée et de la lecture de l'œuvre intégrale puisqu'elle suppose une rencontre directe de l'élève avec l'œuvre. C'est pourquoi, sans exclure les œuvres du programme, on l'associe à une liste d'œuvres de littérature jeunesse régulièrement mise à jour. Cependant elle reste soumise à des exigences portant sur le nombre d'ouvrages et la notation. Seuls trois élèves de $3^{e}$ disent avoir choisi leur livre, mais précisent que c'était dans la limite des 4 livres imposés: l'une parce qu'il était court, une autre parce qu'elle a été attirée par le titre et la première de couverture, la troisième parce qu'il lui restait deux livres à lire et qu'elle a choisi celui qui « l'inspirait le plus». Pour le gros de l'effectif, 7 "non» dont un en majuscules, diverses formulations qui insistent sur l'obligation, l'imposition, l'empiètement sur le temps personnel, - c'est un DM (devoir fait à la maison) -, confirment que l'exercice est considéré à la fois comme scolaire et comme supplémentaire.

La question 8 portait sur les circonstances de l'association objet-livre. Parmi les possibilités évoquées, c'est la $1:$ «pendant la lecture du livre » qui a obtenu le plus de réponses : $11\left(5\right.$ sur 8 en $\left.6^{\mathrm{e}}\right)$. La $2:$ « après, en y repensant » a obtenu 6 réponses (plus du quart de l'effectif). La 3 : «en voyant un objet qui a fait penser au livre » a été cochée par 4 élèves. La $4:$ "à cause d'un passage du livre qui parlait d'un objet semblable » seulement par 2. La 5 : «parce que cet objet paraissait être le portrait du livre » a obtenu autant de réponses que la 2, soit 6 . La consigne a vectorisé la lecture pour la plupart, et pour certains la relecture, que ce soit pour chercher une idée d'objet ou pour vérifier celle qu'ils avaient. La proposition 5 semble correspondre à un critère d'évaluation qui a fixé le choix : elle est couplée aussi bien avec la 1 qui insiste sur la lecture, qu'avec la 3, qui insiste sur l'objet.

Trois élèves ont donné des réponses non prévues, une pour se plaindre qu'elle ne trouvait rien qui correspondait à sa démarche, une pour dire qu'elle avait pensé à l'objet avant de commencer à lire, une troisième pour préciser que ce n'est pas l'objet, mais un tutoriel découvert sur Pinterest qui lui avait donné l'idée de réaliser un lapbook (livre-objet qui regroupe des informations sur un sujet grâce à des images, des dessins, des tableaux et des écrits). Cette dernière réponse pose le problème du statut ontologique de l'objet, dont l'épaisseur sémantique ne se traduit pas forcément par un volume palpable mais aussi bien par une arborescence numérique, le volume devenant alors celui des connexions réalisées entre les informations mobilisées pour le décrire.

Précisément la question suivante proposait un choix de modalités de réalisation : avec un tutoriel internet, un mode d'emploi, le conseil d'un expert, en transformant un objet existant, en réfléchissant tout seul. Tous les élèves ont coché la dernière case, preuve qu'ils ont vraiment eu l'impression d'être des auteurs, même si 8 ont détourné un objet 
existant. Aucun n'a mentionné d'expert, un seul a suivi un mode d'emploi. Celle qui avait suivi Pinterest ne l'a pas mentionné dans la colonne tutoriel internet.

Les questions suivantes portaient sur l'évaluation. La première revenait sur les modalités orale ou écrite et le contenu de la présentation (livre, objet, lecture, fabrication). Les seuls à avoir remis un document écrit pour l'évaluation étaient les élèves de $6^{\text {e }}$. Tous ont parlé du livre, trois de leur lecture, un seul de la fabrication de l'objet. Les $4^{\mathrm{e}}$ et les $3^{\mathrm{e}}$ remettaient l'objet à l'enseignante qui ne donnait de publicité à la présentation que s'ils le souhaitaient. Toutefois une élève de $3^{\mathrm{e}}$ précise que si elle n'a pas présenté son travail en classe, des personnes l'ont vu. On discerne donc deux types d'évaluation, d'un côté l'évaluation scolaire et perçue comme telle, c'est-à-dire uniquement par rapport à la lecture, de l'autre l'évaluation sociale.

question suivante portait sur les réactions de l'enseignant et des camarades. Elle a révélé que l'évaluation informelle de l'objet avait en fait concerné tout le monde. Tous ont répondu sauf une élève (déçue qu'une de ses camarades ait révélé l'identité de son objet avant qu'elle ne l'apporte en classe). Parmi les contenus qui ont provoqué les réactions l'objet tient la vedette (12 voix). Le livre a été ignoré, les idées sur le livre retenues pour 4 élèves, les rapports entre le livre et l'objet pour 5 , et la fabrication de l'objet pour 2. Les couplages indiquent que l'échange conversationnel qui a suivi s'est orienté tantôt sur le rapport à la lecture, tantôt sur la fabrication de l'objet mais pas sur le processus complet.

La question 12 analysait le ressenti des élèves suite aux réactions. Tous, sauf une élève de $4^{\mathrm{e}}$ sans réponse depuis la question 9 , ont dit qu'ils avaient été encouragés, à l'exception de celle dont on avait éventé le secret. Les options destinées à recentrer ce sentiment sur notre problématique de recherche n'ont obtenu que 5 réponses : 0 pour " elles vous ont fait comprendre des choses sur le livre que vous ne compreniez pas auparavant ", 3 pour "donné des idées nouvelles pour fabriquer des objets ", 2 dont une corrélée à l'option précédente pour "donné des idées nouvelles sur le rapport entre le livre et l'objet». L'activité a donc instauré une ambiance festive, mais peu de retours sur la pratique.

L'évaluation était le but de la question 13: "avez-vous été noté, si oui, la note ressemblait-elle aux autres notes de français? », et de la question 14 : «avez-vous déjà fait une fiche de lecture, si oui quelle différence trouvez-vous entre la fiche de lecture et la fabrication de l'objet?». Tous reconnaissent avoir été notés ou devoir l'être. Ceux qui ont eu leur note reconnaissent qu'elle était meilleure que leurs «vraies notes » de français, même pour les bons élèves, sauf pour un cas, sur lequel nous reviendrons. Huit élèves, de $6^{\mathrm{e}}$ mais aussi de $3^{\mathrm{e}}$, ont affirmé n'avoir jamais fait de fiche de lecture. Onze l'avaient fait. Parmi eux 9 préfèrent la fabrication de l'objet à la fiche. Trois champs lexicaux reviennent dans leurs réponses : l'intérêt («motivante, intéressant, agréable, sympa »), le jeu ("amusant, ludique, distrayant» et la créativité (" exprimer notre créativité, faire parler notre imagination, recherche d'un objet original»). Deux réponses sont plus réservées. L'une : "plus de travail», insiste sur le cout de revient, argument qu'on retrouve contré dans une réponse favorable : « ça prend plus de temps mais c'est plus agréable que seulement des fiches de lecture ou des réponses à des questions ». L'autre : "la fabrication est plus difficile que la rédaction » indique un bouleversement d'habitudes chez un "bon élève" dont nous allons découvrir les conséquences. 

travers l'activité. À la question 15 : «cet exercice vous rappelle-t-il ce que vous faites dans d'autres matières?" ", nous avons obtenu plus de réponses attendues : 8 , pour l'Art ou les arts plastiques que pour la précédente activité. Les justifications évoquent la réalisation de projets, la création d'objets («sculptures, affiches »), et même le souvenir d'une pratique interartiale: «car on devait regarder une image et faire un objet en rapport avec cette image ». Un excès de centration sur l'objet a fait dévier sur l'objet intradisciplinaire : « la rédaction » pour l'élève en perte de repères, ou au contraire a incité à l'exclure des apprentissages scolaires : «je trouve que ce travail ne ressemble à aucun autre, il est unique ».

81 La question 16 s'inquiétait du sort du livre et de l'objet. Nous avions prévu que le livre soit rendu ou rangé. Tous l'avaient emprunté à la bibliothèque du défi lecture et tous, sauf deux étourdis, l'avaient rendu. Les sorts envisagés pour l'objet étaient: «rangé bien en vue », « donné », « jeté ", « oublié ». Personne ne l'a jeté. Il a été oublié par 4 élèves, dont un qui avait aussi oublié de rendre son livre. Neuf l'ont posé bien en vue chez eux, réponse dont se rapproche une réponse libre: "rangé dans un endroit approprié ", qui indique une volonté de préserver son jardin secret dans l'espace familial. Sept l'ont donné, deux précisent à qui : «le professeur», une dit: «la professeur l'a gardé ». Nous reviendrons infra sur l'évolution de la relation entre élèves et professeur au cours de l'activité. Mais l'évaluation permet déjà d'esquisser la problématique sous-jacente: l'exercice est-il perçu par les deux parties comme un potlach : un cadeau contre une bonne note, une relation de commanditaire à artiste, ou une relation explicitement didactique ? et dans quelles proportions?

dernière question : «avez-vous recommencé cette expérience tout seul ? si oui, avec quel livre et quel objet? " portait sur un éventuel prolongement privé de l'exercice. Les deux tiers de l'effectif ne l'ont pas envisagé, avec un refus parfois marqué, ce qui indique que l'activité, malgré tout, reste attachée au contexte scolaire. Dans le tiers restant, on notera, à titre indicatif, la présence de 3 des 4 garçons de l'effectif total. Les ouvrages choisis ont été soit des livres imposés qui restaient à lire : Voyage à Itchipoi de J.-C. Moscovici, Zouck de P.Bottero, la saga U4 de J.-L. Blondel, soit des lectures personnelles. Un classique : Harry Potter, pour une élève qui avoue rétrospectivement : «Je l'avais déjà fait avant ou du moins essayé avant avec le premier Harry Potter ». Et Prisonnier de l'overworld, de I. Le Hen (2017), un ouvrage récent choisi par l'élève qui avait été pénalisé par le passage de la rédaction à la fabrication. Cet escape book combine le livre dont vous êtes un héros avec l'escape game (qui consiste à enfermer les joueurs dans un lieu dont ils ne peuvent sortir qu'en résolvant des énigmes, et se joue aussi bien en site naturel que sur site internet : en l'occurrence Minecraft). L'interartialité entre l'édition traditionnelle et les sites de jeu vidéo, en pleine expansion, héroïse les joueurs en mettant leurs aventures en scène, ce qui explique sans doute l'intérêt des garçons, réputés plus joueurs que les filles.

83 aussi « un énorme cube 3D avec sur chaque face une écriture ", symbolisant le rôle du jeu dans Prisonnier de l'overworld et preuve que l'élève désarçonné par la nouvelle consigne l'a prise comme un défi personnel et se l'est appropriée. Une tour Eiffel détournée évoque le rendez-vous parisien dans U4. Des mouchoirs réalisés à la suite de la lecture d'Harry Potter à l'école des sorciers, on ne sait rien, mais ils sont peut-être un 
hommage au vaste mouchoir à pois dans lequel Hagrid enfouit son chagrin en pensant au meurtre des parents d'Harry.

Comme l'enseignant et l'auteur pour l'activité précédente, les enseignantes avaient accepté de répondre à un questionnaire. Nous avons analysé leurs réponses avec le même schéma comparatif, axé sur l'actualisation de la pratique sociale de référence, l'interartialité et les conséquences sur les apprentissages.

Les enseignantes ont débuté cette expérience il y a 5 ans pour l'une (50 ans, 23 ans d'ancienneté dans le métier, dont 14 dans le poste), l'année scolaire 2016-17 pour l'autre (42 ans, 17 ans d'ancienneté dont 15 dans le poste). La pratique sociale de référence n'a eu aucune part dans leur choix. Celui-ci a été guidé essentiellement par une préoccupation professionnelle: trouver un substitut à la fiche de lecture. Cette préoccupation est apparue d'abord chez la première à l'occasion de l'encadrement d'une stagiaire : «nous cherchions des idées novatrices afin de rendre la restitution de la lecture plus ludique pour l'élève et moins ennuyeuse à corriger pour nous. Nous en avions assez de corriger des résumés de lecture copiés sur internet ", décision commune qui a marqué le début d'une relation suivie. Elles mènent actuellement un projet "Correspondances » avec leurs classes de $4^{\mathrm{e}}$ qui « s'écrivent des lettres, lisent des œuvres communes, et se rencontrent 2 fois/an pour mettre en place des ateliers sur diverses pratiques innovantes d'enseignement de la lecture littéraire, en particulier les "Carnets de lecture ».

Excédée elle aussi par les copiés-collés sur internet, la seconde a sauté le pas sous l'effet de plusieurs facteurs: "une idée personnelle qu'elle avait depuis un moment», un stage de formation auquel elle avait assisté, une discussion avec sa collègue et la consultation d'un site internet où des instituteurs montraient leurs réalisations et leurs démarches en primaire. Ni l'une ni l'autre n'ont cobayé la consigne avant de la donner.

Le trajet qui a conduit à la création de ces objets-emblèmes de la lecture sort du schéma de transposition top-down habituel de la pratique sociale de référence. Il est le produit exclusif de la mémoire du métier enseignant et des controverses consécutives à la mise en place de la lecture cursive et de son évaluation ${ }^{6}$. On pourrait le définir comme le passage de l'objet-livre au livre-objet. Dans le primaire, l'entrée dans la lecture littéraire commence par l'objet-livre (cf. l'album de S. Baumann : Qui a fait mon livre?, 2010). Puis l'exploitation de la première et la quatrième de couverture, qui se poursuit au collège, permet de passer du livre objet au livre-lecture. C'est l'introduction de la littérature au cycle 3 en 2002 qui a lancé les controverses sur la lecture cursive, et la façon d'introduire les élèves au plaisir du texte en gardant un juste équilibre entre lecture subjective et lecture distanciée. On a alors vu apparaitre le carnet de lecture et le journal du lecteur. Ce carnet-journal est un objet qui conserve les traces de toutes les lectures, scolaires, périscolaires et privées, et donc de l'histoire du lecteur. Et comme c'est lui qui lui donne forme, il mérite le nom d'objet-livre ${ }^{7}$.

Les documents institutionnels ou professionnels affichent tous la même volonté de faire produire au lecteur un objet qui fonctionne comme un miroir du livre, une sorte de traduction-retour créative, qui n'est pas qu'un métatexte mais possède aussi certaines des qualités esthétiques du texte lu. Il nous semble toutefois que la pratique que nous tentons de décrire ici est un peu différente, en ce qu'elle passe de l'objetcarnet de lecture à un objet-emblème satellite du carnet mais qui a sa propre existence, peut être exposé séparément, pour lui-même, et dont l'apparition n'est pas aussi prévisible, ce qui le rapproche de l'objet d'art. 

émotion et une impatience marqueurs typiques de l'expérience esthétique. À la question: "Quel est l'objet fabriqué dans ce cadre qui vous a le plus marquée?», la première répond : « cette année, une élève de $3^{\mathrm{e}}$ a réalisé un carnet de lecture sur BriseGlace et est arrivée en classe avec une boite dans laquelle elle avait mis son carnet de lecture plastifié dans un gros glaçon [...] qui a tenu jusqu'à chez moi ». La seconde décrit cinq objets, dont un pas encore vu («mais je me régale à l'avance»), entre lesquels elle se refuse de choisir: "parce que je les aime tous». Elle se dit aussi heureuse " que la lecture débouche sur autre chose que l'écrit, pour une fois ", ravie de découvrir «les compétences des élèves que je ne vois pas dans ma discipline habituellement ». Les deux enseignantes ont discuté avec leur collègue d'arts plastiques de certaines des réalisations de leurs élèves. On peut donc dire qu'une certaine prise de conscience s'est opérée quant à la possibilité de co-gérer l'activité avec une autre discipline.

Des conséquences sur les apprentissages, les enseignantes ont surtout développé celles qui concernaient la lecture, en signalant l'amélioration de l'implication des élèves. Celle qui débute dans l'activité se méfie encore de l'objet car elle « pense que l'objet peut être réalisé en ayant lu très partiellement le livre. C'est une des raisons pour lesquelles je ne me contente pas de l'objet mais je demande aussi les ressentis ». Sur les compétences liées à la fabrication de l'objet, leur diagnostic est plus limité. Parmi les compétences proposées comme visées par l'activité, elles classent la compétence algorithmique, la production et la compréhension technologiques loin derrière la compréhension et la production écrite et la maitrise de la langue. De ces apprentissages artistiques cachés, elles retiennent surtout leur brusque apparition, lors de la présentation des objets, et les réfèrent, comme le font les élèves, un peu aux apprentissages en arts plastiques, et beaucoup aux savoirs d'expérience (sans les nommer ainsi) acquis au cours de la fabrication. Pour cette raison, et aussi parce qu'elles craignent de blesser le narcissisme des élèves, elles évitent d'évaluer, ou de faire évaluer les œuvres en classe, si bien que les récits d'expérience, lorsque certains élèves en font, ne débouchent pas sur l'interrogation des pratiques.

91 Pourtant un genre est né, et gagne peu à peu des adeptes dans l'établissement. Comment ? C'est la collègue la plus ancienne dans l'activité qui lève le voile quand, à la question: "Y a-t-il un moment où on compare les objets?", elle répond: "Oui. Les élèves m'offrent certaines de leurs productions et je les montre d'une année sur l'autre aux élèves ». Un rituel qui rejoint celui de la pratique sociale de référence; un salon des beaux-arts miniature qui joue le même rôle que les grands: inciter les nouveaux artistes à rivaliser avec les anciens.

De son côté, la nouvelle venue, qui a maintenu l'évaluation écrite à côté de l'évaluation plastique de la lecture, est en mesure de les comparer. Elle décèle une différence non de degré, mais de nature de l'investissement : « certains élèves s'investissent aussi dans les ressentis. Ils rendent des explications très détaillées. Mais je ne sens pas chez eux ce même plaisir à rendre le travail que pour ceux qui rendent l'objet et qui ont vraiment envie que je sois... ÉPATEE. Je pense qu'ils ont l'impression d'avoir relevé un défi ». Ce miracle : la transformation du contrat didactique en contrat esthétique, est dû au strict cloisonnement entre le plaisir (l'objet) et le devoir (le compte-rendu). Mais il pose le problème - précisément parce qu'il l'évite -, de la didactisation et de l'évaluation de la créativité. 
Enfin, ce travail était un devoir à la maison. Quel rôle jouaient les parents? Pour les enseignantes, il va de soi : «Le travail est donné à la maison, ils peuvent donc se faire aider ». L'enseignante qui pratique l'activité depuis longtemps insiste sur l'étayage des compétences langagières: orthographe et lecture. L'autre sur le support logistique : "plusieurs parents m'ont dit qu'ils avaient aidé à la réalisation, mais que les idées venaient de leurs enfants. Un parent a mis au feu l'objet et l'a retiré du feu (une voiture en carton dans le roman, le père des enfants mourait car sa voiture avait pris feu) [...] un papa et une maman ont prêté leurs voix pour un enregistrement sous forme de JT pour lancer l'enquête policière sur le roman Le professeur a disparu; des parents ont filmé leur enfant pour une vidéo ».

Objets de polémiques récurrentes et accusés de perpétuer les inégalités sociales, les devoirs à la maison, ainsi entendus, deviennent une occasion d'alliance éducative, dont l'élève reste le maitre d'œuvre, et sont un facteur puissant de rapprochement de l'école et des familles autour d'un travail qui est aussi une œuvre dont tous peuvent s'enorgueillir.

\section{Conclusion}

Nous avions choisi ces deux activités pour leur complémentarité théorique, mais l'analyse des données a montré qu'elles avaient un point commun. Comme la plupart des activités du français, elles reposent sur une exploitation unilatérale des arts au service des apprentissages langagiers et littéraires. Ce qui, pour des apprentissages interartiaux, qu'elles ne visaient ni l'une ni l'autre, constitue une faiblesse.

En revanche, nous avons découvert qu'elles présentaient une autre forme de complémentarité : dans leurs rapports aux pratiques artistiques de référence. L'une repose sur une stratégie d'étayage réciproque de l'écrivain et de l'enseignant, pour le plus grand bénéfice des élèves, interpellés dans leur expertise de lecteurs réels, et encouragés dans leur apprentissage de scripteurs. L'autre est le fruit de la reconception (Espinassy \& Saujat, 2003, p. 111) d'une tâche d'enseignement, qui visait à préserver à la fois l'efficacité de l'enseignement et la réalisation de soi. Trouver du sens aux apprentissages est aussi important - sinon plus - pour l'enseignant que pour les élèves. Nous avons pu constater qu'une situation particulière « dans un contexte qui tend à pousser le métier dans ses retranchements constitue un révélateur de composantes du travail enseignant » (ibid., p. 113) : en l'occurrence l'efficacité, le plaisir au travail et l'entente entre élèves et enseignant; du travail apprenant aussi. Nous avons vu que la plupart des élèves étaient prêts à consentir un effort supplémentaire s'ils y gagnaient la fierté de créer, la reconnaissance du public et la richesse d'une expérience.

La reconception d'une tâche peut parfois déboucher sur une autre tâche. L'enseignement est une démarche de recherche, et comme toute recherche, est soumis à sérendipités. Prendre en charge didactiquement cette nouvelle tâche ou pas, à une ou à plusieurs disciplines, est de la décision des enseignants. Mais cette décision tiendra toujours compte de l'ensemble des composantes du métier, comme l'ont confirmé également les critères d'invitation des écrivains dans la classe.

Pour faire passer la complémentarité décelée entre les deux activités enquêtées du stade de la théorie à celui de la pratique, il faudrait que les mêmes élèves puissent en bénéficier, et que soit mis en place un ancrage interdidactique avec les enseignants 
d'arts plastiques mais aussi de technologie - pour la démarche de projet - ou d'autres disciplines, en fonction des projets. Même si les nouveaux programmes et le nouveau mode d'évaluation par compétences prévoient explicitement ce type d'organisation et que les manuels en donnent des exemples, l'impulsion ne peut venir que des communautés éducatives, après analyse de leurs forces, de leurs faiblesses et de leurs capacités d'engagement.

Pour un enseignant de français, l'apprentissage de la langue, des discours et de la littérature est prioritaire. Cependant la découverte de l'épanouissement procuré aux élèves par la création artistique, ou par la discussion d'égal à égal avec un écrivain, peut être une raison suffisante pour qu'il accepte de se risquer dans la collaboration interdidactique tous azimuths, préconisée par les programmes. Nous avons vu dans la première partie de notre étude que l'histoire de sa discipline l'a plutôt habitué à occuper une position dominante par rapport aux arts et à l'histoire, intégrés à très petites doses dans les manuels et dans les enseignements, et adaptés aux besoins de la discipline. Par ailleurs le stéréotype encore très actif des familles de disciplines a pu lui faire oublier que les compétences réelles exigées par les arts (littérature comprise) franchissent allégrement la frontière entre disciplines "littéraires" et disciplines "scientifiques ». L'architecture se sert de la géométrie et de la physique, la peinture de la géométrie et la chimie, et le roman d'à peu près tout.

Le dernier obstacle à examiner est donc celui de la motivation. À l'issue de notre première partie nous nous demandions si l'image ne risquait pas de détourner l'intérêt du texte littéraire au lieu d'en faciliter la lecture. Nous pouvons nous demander maintenant si développer une interdidactique des arts et de la littérature ne risque pas de transformer le plaisir de la création artistique et la détente qu'elle apporte dans la classe en un nouveau pensum, et donc de nuire aussi à la lecture littéraire, mais par une autre voie. Comment éviter la formation de ce cercle infernal ?

D'abord en dénouant la malédiction originelle qui pèse sur la didactique de l'écriture créative, celle de sa subordination à la lecture littéraire, qui, si elle a eu l'avantage d'introduire, comme l'a montré l'ensemble de notre étude, les pratiques interartiales dans les apprentissages, en limite l'exercice et la prise de conscience. Dans un article qui date de 1996, É. Louis (1996, p. 154) alertait déjà sur les dangers d'une écriture qui ne serait que le prolongement de la lecture: «ce n'est pas exagéré de dire qu'alors l'écrit risque de ne plus être que l'inscription du travail de lecture, et que l'évaluation critériée du texte-cible peut ne plus apparaitre que comme un contrôle de la lecture du texte-source ». Et nous reprenons volontiers à notre compte son affirmation : « je crois que l'écriture doit aussi être enseignée comme une expérience, si l'on veut que les élèves y recourent spontanément » (ibid.).

102 À l'époque, le cahier d'écrivain (Seweryn \& Bucheton, 1999), dans lequel l'élève écrivait ou réécrivait à sa guise un certain nombre de textes par semaine à partir de lanceurs proposés en classe et négociés avec l'enseignant, était considéré comme le support par excellence de l'écriture créative individuelle. Il complétait l'atelier d'écriture, réservé aux apprentissages communs des techniques d'écriture et aux projets collectifs. Ces deux dispositifs relevaient d'un modèle d'enseignement basé sur l'expérience et non sur les savoirs théoriques, même s'il faisait appel aux savoirs grammaticaux ou culturels en fonction des besoins rencontrés. Ils visaient (déjà !) la construction de compétences : 
L'acte de travail devient acte de formation lorsqu'il s'accompagne d'une activité d'analyse, d'étude ou de recherche sur lui-même. La production de savoir par l'apprenant est alors utilisée comme un outil direct de production de compétence, alors que traditionnellement dans les systèmes d'enseignement et de formation, les transformations identitaires souhaitées sont considérées comme devant être obtenues pour l'essentiel soit par mise à disposition - appropriation des savoirs extérieurs, soit par mise en exercices des savoirs appropriés. (Barbier, 1996, p. 3).

Le type de recherche que nous venons d'exposer participe également de cette construction, en incitant les acteurs à analyser leurs pratiques.

C'est donc sur une reconception du double dispositif, atelier d'écriture et carnet d'écrivain, que nous proposons d'appuyer la didactisation des pratiques interartiales. Cela n'exige pas de supprimer le rapport aux œuvres (les marges des manuscrits des grands écrivains sont remplies des citations de leurs lectures, et Montaigne écrivait à partir de citations). Il faut juste oser quitter le point de vue du lecteur et prendre celui de l'auteur qui n'hésite pas à puiser son inspiration partout où il la trouve, dans les œuvres littéraires, les œuvres empruntées aux arts, et tout ce qui dans son environnement lui parait digne d'être le support d'une émotion à faire partager.

Ajoutons, même si cela va sans dire, qu'un tel cahier de création doit être ouvert aux pratiques intermédiales quotidiennes à la fois pour profiter de leurs ressources qui permettent de passer de l'image à la musique et au texte, ou du dictionnaire à l'écrit, et à son courrier électronique en un effleurement, et pour infléchir leurs usages vers des pratiques plus réflexives et plus novatrices. Nous avons la chance que cette intermédialité quotidienne mette la pratique interartiale à la portée de tous, il faut savoir la saisir.

Nous laisserons le dernier mot de cette étude à l'emblème inventé par une élève, cet objet éphémère formé par un glaçon qui fond pour libérer un carnet de lecture imperméabilisé, en espérant qu'il alertera à la fois sur l'imperméabilité de l'école et sur l'indéfectible capacité de ses acteurs à la surmonter.

DOQUET-LACOSTE, C. (2008). «Carnet de lecture, un espace d'émergence de la lecture littéraire ». Argos 43, p. 51-54. En ligne: http://www.educ-revues.fr/ARGOS/AffichageDocument.aspx? iddoc $=37493$.

\section{BIBLIOGRAPHIE}

\section{Textes officiels}

DIRECTION DE L'ENSEIGNEMENT SCOLAIRE. (1999). Enseigner au collège. Français. Programmes et accompagnement. Paris : Centre national de documentation pédagogique.

MiNiSTÈRE DE L'ÉDUCATION NATIONALE. B.O. spécial $\mathrm{n}^{\circ} 6 \mathrm{du} 20$ août 2008, Programmes du collège, Programmes de l'enseignement du français $\left(2009-10: 6^{\mathrm{e}} ; 2010-11: 5^{\mathrm{e}}, 2011-12: 4^{\mathrm{e}} ; 2012-13: 3^{\mathrm{e}}\right)$. 
Le Parcours d'Éducation Artistique et Culturelle, arrêté du $1^{\text {er }}$ juillet 2015. En ligne : http:// eduscol.education.fr/cid74945/le-parcours-education-artistique-culturelle.html.

MINISTÈRE DE L'ÉDUCATION NATIONALE. (2016). Document d'accompagnement pour l'évaluation des acquis du socle commun de connaissances, de compétences et de culture. Éléments pour l'appréciation du niveau de maîtrise satisfaisant en fin de cycle 4. En ligne : eduscol.education.fr/ ressources-2016.

\section{Manuels}

ARNAUD, L. \& GARRIGUE, A. (1982). Mots et Merveilles 5 ${ }^{e}$. Paris : Magnard. BeLtRANDO, B. (dir) (2016). Français $6^{e}$. Livre unique. Paris : Hatier.

BERTAGNA, C. \& CARRIER, F. (dirs) (2016a). Français $4^{e}$. Vanves : Hachette. BERTAGNA, C. \& CARRIER, F. (dirs) (2016b). Français $3^{e}$. Vanves : Hachette. BESSON, J.-J., BASTARD-GALLET, S. \& RAYMOND, M.-T. (2001). Texto collège $5^{e}$. Le français en séquences. Paris : Hachette.

BRIAT, C. et al. (2011). Français $4^{e}$. Paris : Magnard.

LAGACHE, F. et al. (2010). Français $5^{e}$. Paris : Belin.

LAGARDE, A. \& MICHARD, L. (1967) [1951]. XVII e siècle. Paris : Bordas.

POTELET, H. (dir.) (2014). Français $6^{e}$. Paris : Hatier.

RANDANNE, F. (dir.) (2016). Français $4^{e}$. Paris : Belin.

BALDNER, J.-M. \& BARBAZA, A. (2013). « Histoire des arts : de la notion à la discipline ». Le français aujourd'hui 182, p. 3-9. En ligne : https://www.cairn.info/revue-le-francais-aujourd-hui-2013-3page-3.htm.

BARBIER, J.-M. (1996). «Introduction ». In : Barbier, J.-M. (dir.), Savoirs théoriques et savoirs d'action. Paris : Presses universitaires de France, p. 1-13.

BIAGIOLI, N. (2013). «Lecture de l'image et histoire des arts : des ressources pour mettre en place une compétence culturelle scolaire ? ». Actes du $3^{e}$ Colloque international de l'ARCD. En ligne : https:// hal.archives-ouvertes.fr/hal-00757216V1.

BIAGIOLI, N. (2016). «Ce qui est vivant et ce qui est mort de la didactique de l'écriture littéraire ?» In : Petitjean, A. (dir.), Didactiques du français et de la littérature. Metz : Crem, Université de Lorraine, p. 599-617.

BIAGIOLI, N. \& TORTERAT, F. (2012). « La recherche en interdidactique : apports méthodologiques et pratiques ». In : Elalouf, M.-L., Robert, A., Belhadjin, A., \& Bishop, M.-F. (dirs.), Les didactiques en question(s). Bruxelles : De Boeck, p. 269-278.

BOISSINOT, A. \& HOUDART-MÉROT, V. (2001). « Vers de nouveaux équilibres : de 1975 à nos jours ». In : Boissinot, A., Armand, A. \& Jordy, J. (coords), Le français en collège et en lycée. Paris : Hachette, p. 36-42.

BRICCO, E. \& MURZILLI, N. (2012). «Introduction ». Cahiers de narratologie 23. En ligne : https:// narratologie.revues.org/6639.

BUTOR, M. (1969). Les mots dans la peinture. Genève : A. Skira. 
CHABANNE, J.-C. (2016). «Langue, littérature, art(s), langage(s) : didactique de la littérature et didactiques des arts, un dialogue fécond à entretenir et à renouveler ». In : Petitjean, A. (dir.), Didactiques du français et de la littérature. Metz : Crem, Université de Lorraine, p. 157-176.

CLAUDE, M.-S. (2016). «Commentaire de la peinture et commentaire de la littérature : étude comparée de l'activité des élèves ». In : Petitjean, A. (dir.), Didactiques du français et de la littérature. Metz: Crem, Université de Lorraine, p. 561-580.

COHEN-AZRIA, C. (2010) [2007]. « Pratique sociale de référence ». In : Reuter, Y. (dir.), Dictionnaire des concepts fondamentaux des didactiques. Bruxelles : De Boeck, p. 179-182.

DOZ, C. (2014). « La lecture cursive et le journal du lecteur ». En ligne : https://fr.Slideshare.net/ ClaireDoz/la-lecture-cursive.pp.

ESPINASSY, L, SAUJAT, F. (2003). «Enseigner les arts plastiques en ZEP : les dessous du métier ».

Recherche et formation 44, p. 111-124. En ligne : http://www.persee.fr/doc/

refor_0988-1824_2003_num844_181872.

FAYOL, M. (1996). «La production du langage écrit ». In : David, J. \& Plane, S. (dirs), L'apprentissage de l'écriture de l'école au collège. Paris, Presses universitaires de France, p. 9-36.

GIRAUDO, L. (2006). Michel Butor, le dialogue avec les arts. Villeneuve d'Ascq : Presses universitaires du Septentrion.

JOOLE, P (2010). «Quoi écrire dans un carnet de lecture? ", Actes des $11^{e}$ rencontres des chercheurs en didactique de la littérature. En ligne : http://www.unige.ch/litteratures2010/contributions_files/ joole2010.pdf.

LACELLE, N. (2009). Modèle de lecture-spectature, à intention didactique, de l'œeuvre littéraire et de son adaptation filmique. Thèse : Université du Québec à Montréal. En ligne : http:// www.archipel.uqam.ca/view/divisions/educ/2009.html.

LAHIRE, B. (2006). La condition littéraire. La double vie des écrivains. Paris : La Découverte.

LANGLADE, G. (2004). « Sortir du formalisme, accueillir des lecteurs réels ». Le français aujourd'hui 145, p. 85-96. En ligne : https://www.cairn.info/revue-le-francais-aujourd-hui-2004-2-page-85.htm.

LOUIS, É. (1996). «Pratiques d'écriture au collège : tendances nouvelles, nouveaux problèmes ». In : David, J. \& Plane, S. (dirs), L'apprentissage de l'écriture de l'école au collège. Paris : Presses universitaires de France, p. 131-157.

MOSER, W. (2007). «L'interartialité : pour une archéologie de l'intermédialité ». In : Froger, M. \& Müller J. E. (dirs), Intermédialité et socialité. Münster : Nodus, p. 69-92.

ORIOL-BOYER, C. \& DUMINY-SAUZEAU, C. (2016). «Atelier d'écriture littéraire et didactique ». In : Petitjean, A. (dir.), Didactiques du français et de la littérature. Metz : Crem, Université de Lorraine, p. 581-598. REUTER, Y. (2010). «Disciplines scolaires ». In : Reuter, Y. (dir.), Dictionnaire des concepts fondamentaux des didactiques. Bruxelles : De Boeck, p. 85-89.

SCHAEFFER, J.-M. (2015). L'expérience esthétique. Paris : Gallimard.

SERRE-FLOERSHEIM, D. (2001). « L'image dans l'enseignement du français ». In : Boissinot, A., Armand, A. \& Jordy, J. (coords), Le français en collège et en lycée. Paris : Hachette, p. 212-219.

SEWERYN, B. \& BUCHETON, D (1999). «Le cahier d'écrivain : d'un grand nombre d'écrits vint la créativité ! » Le français aujourd'hui 127, p. 73-82. 


\section{NOTES}

1. Ce terme recouvre l'ensemble des relations qui peuvent se produire entre des pratiques artistiques diverses : absorption, recyclage, traduction, citation, développement. En simplifiant, on pourrait dire qu'il s'agit d'une relation d'hypertextualité au sens de G. Genette dans la mesure où l'existence d'une œuvre (hypertexte) dépend d'une ou plusieurs œuvres qui l'ont précédée (hypotexte), mais avec deux clauses supplémentaires : 1) hyper texte et hypotexte appartiennent à des sémiotiques dont le plan de l'expression diffère (ex : musique et peinture); 2) les relations tendent à la réciprocité: les romanciers se mettent à photographier (ex : É. Zola) au contact des photographes et réciproquement (ex: Nadar).

2. La présentation : "Harmonie de la composition, netteté du dessin, prédominance de l'élément humain: avec cette œuvre, notre peinture classique atteint son apogée au moment où le classicisme commence à s'affirmer en littérature ", résume les traits principaux de la culture scolaire du secondaire à l'époque: classicisme nationaliste (refus du baroque européen), humanisme et interartialité, avec une référence encore vivace au règne de Louis XIV comme apogée de la littérature française.

3. «Matière interdidactique » (cf. Biagioli, 2013) parce qu'elle est formée par prélèvement de parties de matières enseignées dans l'ensemble des disciplines du socle, et fait l'objet d'un projet d'enseignement commun spécifique avec ses programmes et ses instructions. C'est certes un projet interdisciplinaire, au sens littéral, puisqu'il concerne plus d'une discipline, mais son objectif n'est pas de faire appliquer à des situations de la vie des savoirs acquis dans différentes disciplines, comme le font les projets dits interdisciplinaires, mais de mobiliser les disciplines autour d'une situation didactique et d'un objet d'enseignement communs (Biagioli \& Torterat, 2012, p. 270).

4. I3DL, Interdidactique, Didactiques des disciplines et des langues, EA 6308.

5. Ex : «Préparer l'organisation de son récit: 1 . Au brouillon, définissez et numérotez les étapes de votre récit [...] 2. Choisissez le narrateur ( $1^{\text {re }}$ ou $3^{\mathrm{e}}$ personne) » (Beltrando, 2016, p. 165).

6. Pour le détail de cet historique, nous renvoyons à $P$. Joole (2010) : «Quoi écrire dans un carnet de lecture", pour celui des controverses professionnelles à G. Langlade (2004): «Sortir du formalisme, accueillir des lecteurs réels» et Cl. Doquet-Lacoste (2008) «Carnet de lecture, un espace d'émergence de la lecture littéraire ».

7. Pour plus de détails, nous renvoyons à C. Doz (2014) « La lecture cursive et le journal du lecteur $»$.

8. Phénomène qui consiste à faire une découverte de façon fortuite, le plus souvent dans le cadre d'une recherche qui concerne un autre sujet (d'après Wikipedia).

\section{RÉSUMÉS}

Dès les premiers manuels de français illustrés, l'image et l'histoire des arts ont été mises au service de l'enseignement de la langue et de la littérature. Depuis que le dialogue des arts et de la littérature - qui suppose l'équilibrage de leur relation -, est devenu un objectif interdidactique, enseignants et élèves ont dû reconsidérer leurs pratiques. L'étude est organisée en deux parties : la première est une présentation des pratiques interartiales dans le contexte scolaire, la seconde analyse deux pratiques de classe en collège : l'écriture d'un texte à partir d'une première de couverture, et la fabrication d'un objet pour rendre compte d'une lecture cursive. 
From the earliest illustrated French textbooks, image and history of the arts were used for teaching language and literature. Since the dialogue between arts and literature - which presupposes the balancing of their relationship - has become an interdidactic goal, teachers and students have had to reconsider their practices. Our study is organized in two parts: the first is a presentation of inter-artial practices in the school context, the second analyzes two classroom practices in college: writing of a text from a front cover, and manufacturing an object to give account of a cursive reading.

\section{INDEX}

Mots-clés : didactique du français, interdidactique, didactique de la littérature, pratique sociale de référence, interartialité

Keywords : didactics of French, interdidactics, didactics of literature, social practice of reference, interartiality

\section{AUTEUR}

\section{NICOLE BIAGIOLI}

Université Nice Sophia Antipolis, I3DL, EA 6308, F-06046, France 